\title{
Evaluation of outcome reporting trends for femoroacetabular impingement syndrome- a systematic review
}

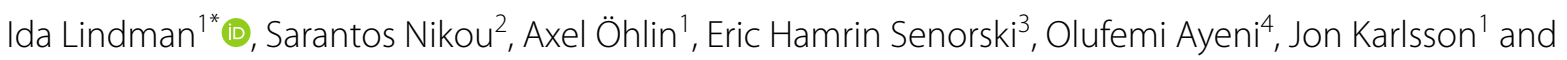 \\ Mikael Sansone ${ }^{1}$
}

\begin{abstract}
Purpose: The aim of this systematic review was to evaluate the trends in the literature regarding surgical treatment for femoroacetabular impingement syndrome (FAIS) and to present which patient-reported outcome-measures (PROMs) and surgical approaches are included.

Methods: This systematic review was conducted with the PRISMA guidelines. The literature search was performed on PubMed and Embase, covering studies from 1999 to 2020. Inclusion criteria were clinical studies with surgical treatment for FAIS, the use of PROMs as evaluation tool and studies in English. Exclusion criteria were studies with patients $<18$ years, cohorts with $<8$ patients, studies with primarily purpose to evaluate other diagnoses than FAIS and studies with radiographs as only outcomes without using PROMs. Data extracted were author, year, surgical intervention, type of study, level of evidence, demographics of included patients, and PROMs.

Results: The initial search yielded 2,559 studies, of which 196 were included. There was an increase of 2,043\% in the number of studies from the first to the last five years (2004-2008) - (2016-2020). There were 135 (69\%) retrospective, 55 (28\%) prospective and 6 (3\%) Randomized Controlled Trials. Level of evidence ranged from I-IV where Level III was most common (44\%). More than half of the studies (58\%) originated from USA. Arthroscopic surgery was the most common surgical treatment (85\%). Mean follow-up was 27.0 months ( \pm 17 SD), (range 1.5-120 months). Between 1-10 PROMs were included, and the modified Harris Hip Score (mHHS) was most commonly used (61\%).
\end{abstract}

Conclusion: There has been a continuous increase in the number of published studies regarding FAIS with the majority evaluating arthroscopic surgery. The mHHS remains being the most commonly used PROM.

Keywords: Femoroacetabular impingement syndrome, FAIS, Patient-reported outcome measures, PROM, Hip arthroscopy

\footnotetext{
*Correspondence: Lindman91@hotmail.com

${ }^{1}$ Department of Orthopaedics, Institute of Clinical Sciences, Sahlgrenska

Academy, University of Gothenburg, 41345 Gothenburg, Sweden

Full list of author information is available at the end of the article
} 


\section{Introduction}

In 1936 Smith-Petersen described hip pain caused by a bone-to-bone impingement between the femoral neck and the acetabulum [196]. However, it was not until 2003 that the modern concept of femoroacetabular impingement was initiated by Ganz et al. [74].

Femoroacetabular impingement syndrome (FAIS) results from an abnormal morphology of either the femoral head (cam) or the acetabulum (pincer) or a combination of both. This causes an incongruence in the hip joint and is a common source of hip pain, especially in the young active population [216]. Surgical treatment of FAIS aims to restore the normal hip joint morphology and thereby reduce symptoms [154]. Open hip dislocation was initially considered the gold standard for surgical treatment of FAIS, however, the use of a minimally invasive approach with arthroscopy has increased during the $2010^{\prime} \mathrm{s}[46,154]$.

With an escalation of the arthroscopic procedures performed, there has been a corresponding increase in the studies published regarding FAIS [106]. Furthermore, several registries have been developed to keep track of performed arthroscopies and evaluate the outcomes after the procedures $[93,126,185]$. Patient-reported outcome measures (PROMs) are commonly used for evaluating the patients' perspective of outcome of surgical treatment [158]. According to the Warwick Agreement, defined in 2016, the Hip and Groin outcome score (HAGOS) [205], Hip Outcome Score (HOS) [134] and the international Hip Outcome Tool (iHOT) $[84,143]$ are recommended as preferable PROMs for evaluating the outcome after FAIS surgery [82]. These PROMs are noted to be valid, reliable and responsive after FAIS surgery [170]. Yet, the PROMs used for FAIS have most commonly been developed for an older patient category with osteoarthritis, such as Harris hip score (HHS), while the PROMs recommended for the younger population are gradually being adopted [206]. With the use of PROMs developed for another patient category or condition, there is a risk of ceiling or wash-out effects due to the inclusion of nonrelevant items.

The aim of this systematic review was to evaluate the trends in the literature pertaining to FAIS. More specifically, the aim was to present trends for the PROMs used and which surgical approaches have been performed to treat patients with FAIS. The hypothesis was that an increase in the number of studies with arthroscopic procedures performed would be observed with the majority using hip specific PROMs.

\section{Methods}

The systematic review was governed in agreement with the Preferred Reporting Items for Systematic Review and Meta-Analysis protocols (PRISMA) [142].

\section{Eligibility criteria}

All inclusion and exclusion criteria were prespecified and designed as recommended by PRISMA. The inclusion criteria for this systematic review were clinical studies with patients undergoing surgical treatment for FAIS. Studies defined as prospective, retrospective and randomized controlled trials (RCTs) were included. Only studies comprising PROMs were included. The study could be either therapeutic or prognostic. Therapeutic studies defined as studies exploring the results of FAIS surgery, and, prognostic studies, defined as investigating the effect of a patients' characteristic on the outcome of FAIS. Only studies with English language in full text were included.

Exclusion criteria were studies including adolescents, children or described as "open physes". No studies with patients $<18$ years were included. Studies with less than 8 patients were deemed not eligible. Studies with primarily patients with slipped capital femoral epiphysis and Leg-Calve-Perthes disease were excluded. Studies with radiographic measurements as only outcomes were also excluded. Conference papers, systematic reviews, commentaries, protocols, narratives and studies validating PROMs were excluded. Studies with primary purpose to evaluate other diagnoses than FAIS and studies with patients undergoing revision surgery were also excluded.

\section{Information sources and search}

A systematic literature search was conducted in the online databases PubMed and Embase in September 2020. The searches were performed by a librarian with expertise in electronical searches at the Sahlgrenska University Hospital Library, Gothenburg, Sweden. The search retrieved studies from the period January 1999 until search day $7^{\text {th }}$ of September 2020 to include an interval of over 20 years. The search was performed with controlled terminology and words. Different variations of the terms for "femoroacetabular impingement" OR "FAI" OR "hip impingement" OR "CAM impingement" OR "Pincer Impingement" were used together with different variations of "surgery" OR "operative" OR "arthroscopy" to create the search string. Exact information about the details on the search strategies for the database PubMed is found in Appendix, (Table 2).

\section{Study selection}

The studies from the electronic search were systematically evaluated by titles, thereafter abstract and finally their full texts by two reviewers (IL and SN). Both reviewers evaluated all studies from both databases independent of each other. Duplicates were removed manually. If the title or the abstract did not provide enough information regarding inclusion, the study was automatically included to the full-text assessment. The two reviewers were not blinded 
to the author, year and journal of publication. After all full texts were independently decided by the two reviewers, any disagreements regarding inclusion of studies were solved with discussion between the two reviewers.

\section{Data items}

The data extracted included the level of evidence, title of the study, authors, year of publication, journal, country where study was performed, type of study (retrospective, prospective, RCT), included number of, and which different PROMs used in the study. The proportion of "hip specific" PROMs in the study was recorded in the extraction sheet. In addition to exploring the development of included PROMs over the years, 2016, when the Warwick agreement was stated, was used as a cut-off to evaluate the adoption of recommended PROMs. It was noted if the study had included any type of "rate of return to sport" (RTS) apart from using a regular PROM and if the study evaluated patient satisfaction. Inclusion of any RTS assessment was in this study defined dichotomously (yes or no). Type of interventions assessed in the study were divided into open, arthroscopic or a combination of arthroscopic/open. Further data as proportion of sex, follow-up time, and number of patients were collected. The number of patients were defined as the patients undergoing surgical intervention, i.e., if the control group consisted of patients without receiving intervention, the control group was not included. Distribution of sex and mean follow-up for the last visit were recorded.

\section{Statistical analyses}

Interobserver agreement for full-texts was calculated with the Cohen kappa coefficient ( $\mathrm{k}$ ) [119]. According to previous recommendations the values of $\kappa$ were set a priori with a $\mathrm{K}$ of $0-0.2$ equals slight agreement, $0.21-0.4$ fair agreement, $0.41-0.6$ moderate agreement, $0.61-0.8$ substantial agreement and $>0.8$ equals to near perfect agreement. Descriptive statistics were used to present the data. Mean, standard deviation (SD), median and range values were presented when appropriate. Follow-up period was presented either as average follow-up period, or if not presented in the study, as minimum follow-up period. For studies comparing two or more groups, and no average follow-up period was mentioned for the entire cohort, a combined average follow-up was calculated. The analyses were performed with Microsoft Excel (version 16.40, Microsoft Corporation).

\section{Results}

\section{Study identification and characteristics}

The first search revealed 2,085 studies in PubMed and 2,218 studies in Embase. After removing duplicates, a total of 2,559 unique studies were eligible for the screening process. Figure 1 displays a flowchart of the screening process in accordance with the PRISMA guidelines. The agreement between the two readers for inclusion of full-text was $97 \%$ with a Cohen kappa value of 0.82 , considered as near perfect agreement.

There were $6(3 \%)$ RCTs, 55 (28\%) prospective studies and 135 (69\%) retrospective studies included in this systematic review. There were $6(3 \%)$ Level I studies, 21 (11\%) Level II studies, 86 (44\%) Level III studies and 83 (42\%) Level IV studies (Table 1). The included studies were published between 2004-2020. There was a large increase of published studies in the latter years where 143 (73\%) of the studies were published in the last 5 years (2016-2020) compared to 7 (4\%) in the first 5 years (2004-2008), an increase of 2,043\% (Fig. 2).

More than half of the studies (58\%) were conducted in USA. Most studies were published in The American Journal of Sports Medicine (21\%), followed by Arthroscopy: The Journal of Arthroscopic and Related Surgery (19\%). A total of 32,303 patients were included counting the patients in all studies together, with an average of 165 patients per study (range 8-1,102). The mean follow-up period was 27.0 months ( $\pm 17 \mathrm{SD})$, (range 1.5-120) (Table 1).

\section{Surgical procedure}

The majority of the included studies (85\%) were evaluating arthroscopic treatment. Only $5 \%$ of the included studies were examining solely open dislocation while the remaining $10 \%$ discussed either both open and arthroscopic or defined a mini-open technique with arthroscopic assistance. The procedure described in each study is reported in Table 1.

\section{Patient-reported outcome measures}

A total of 39 different PROMs were found in the studies, of these, $15(38 \%)$ were hip-specific (Table 3, in Appendix). Between 1-10 PROMs were used in each study with an average of 3 ( $\pm 1.8 \mathrm{SD})$ PROMs per study. Before 2016, the median of included PROMs was two per study, and after 2016 the median had increased to three per study.

The most common used hip-specific PROM was mHHS (used in 120 studies (61\%)), followed by HOS (81 studies (41\%)) (Fig. 3). An additional question of return to sport/return to activity was seen in $13 \%$ of the included studies. Of 196 studies, 40\% included a question on satisfaction of which the majority used the visual analog scale.

During the first five years (2004-2008), the Merle d'Aubigné and Postel score and the Western Ontario 


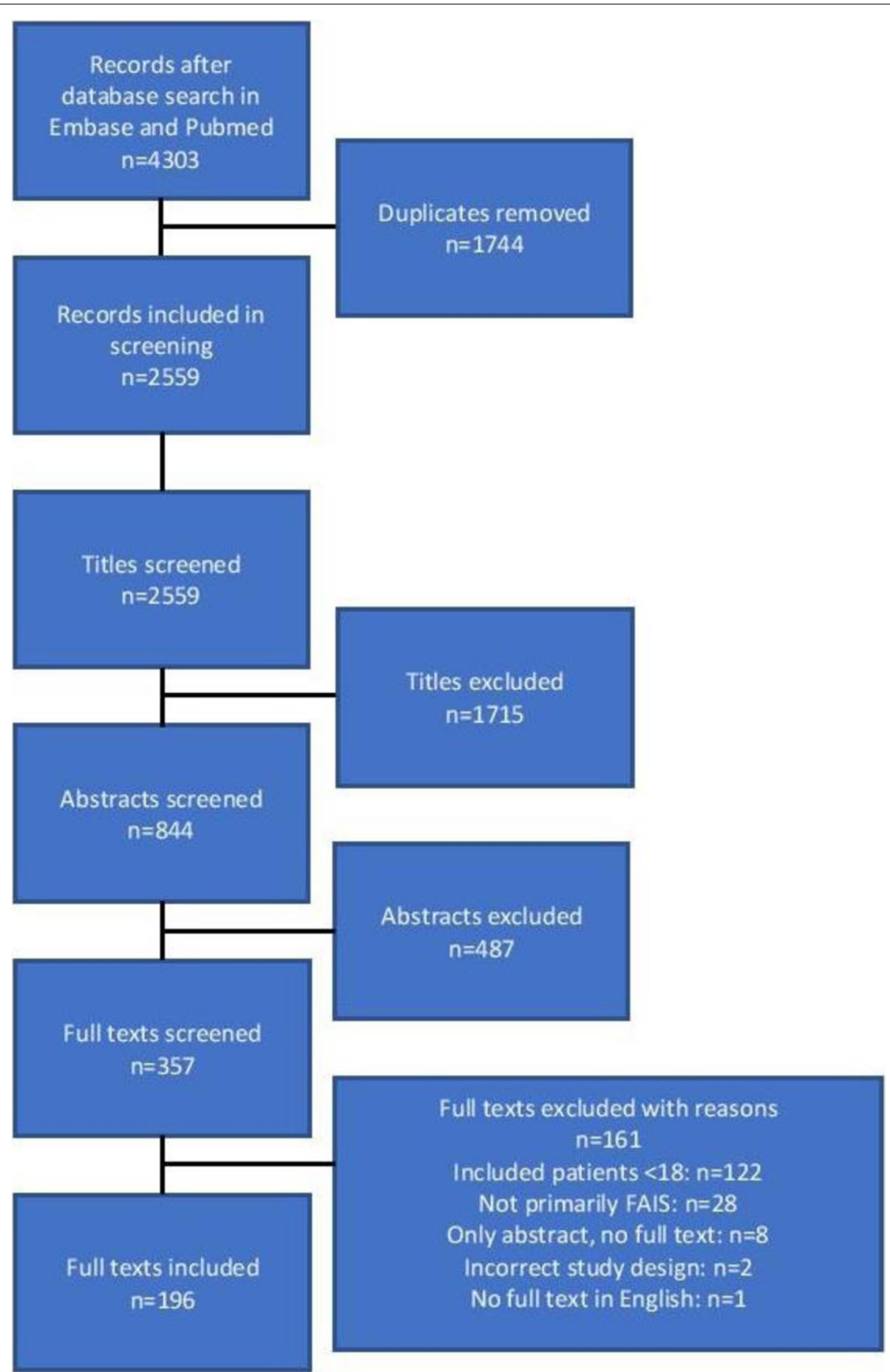

Fig. 1 Flow chart of the screening process and number of included studies

and McMaster Universities Osteoarthritis Index (WOMAC) were equally the most commonly used scores, reported in $3(43 \%)$ of the studies during that period. During the last five years (2016-2020), the mHHS was the most commonly used, in $93(65 \%)$ of the studies.
Of the 143 studies published during or after 2016, 67 (47\%) studies have included the HOS, 46 (32\%) included either iHOT-12 or iHOT-33 and $12(8 \%)$ studies included the HAGOS (Fig. 3). Fifty-two of the 143 studies (36\%) did not use any of the three PROMs recommended by the Warwick agreement [82] (Table 1). 


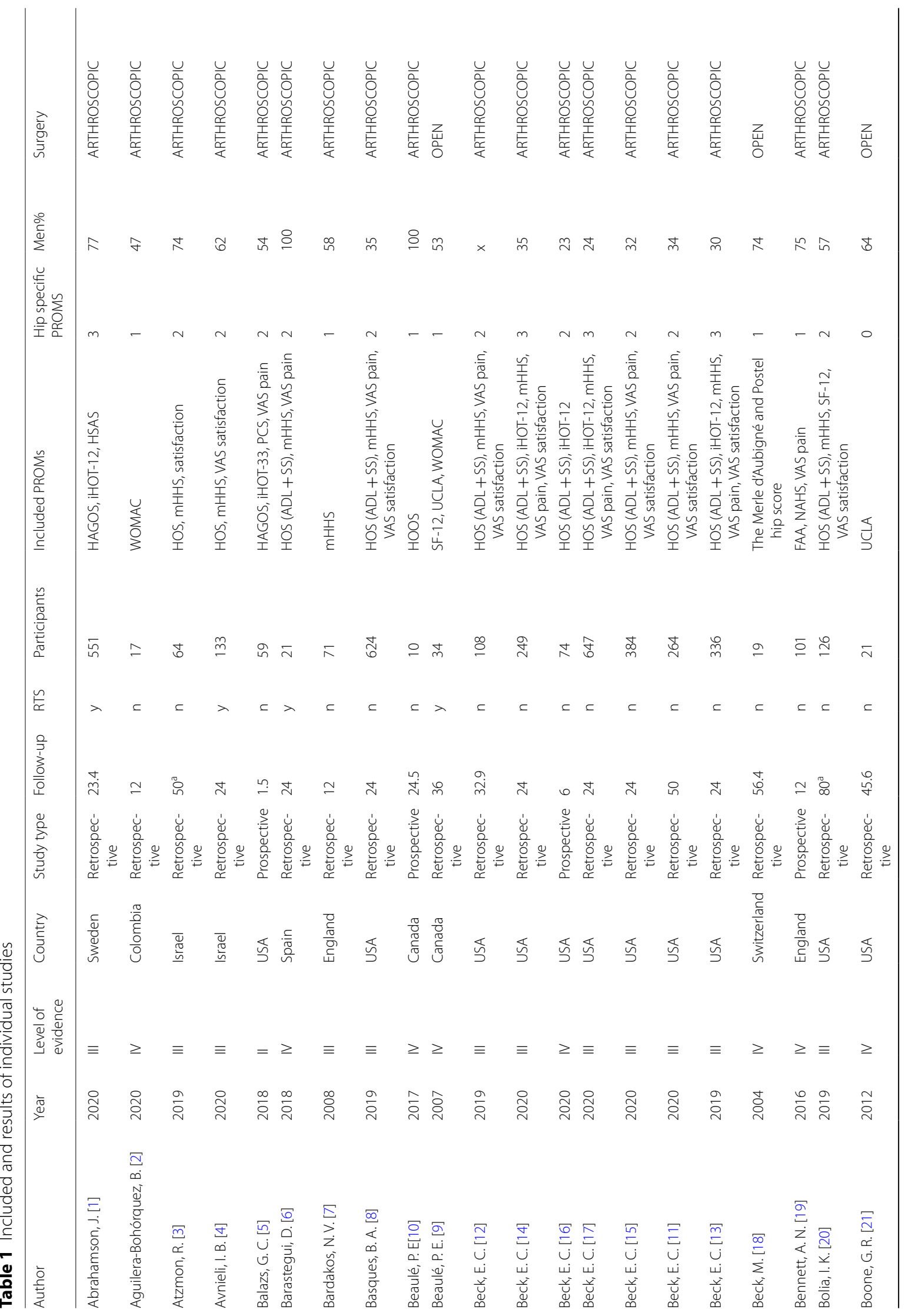




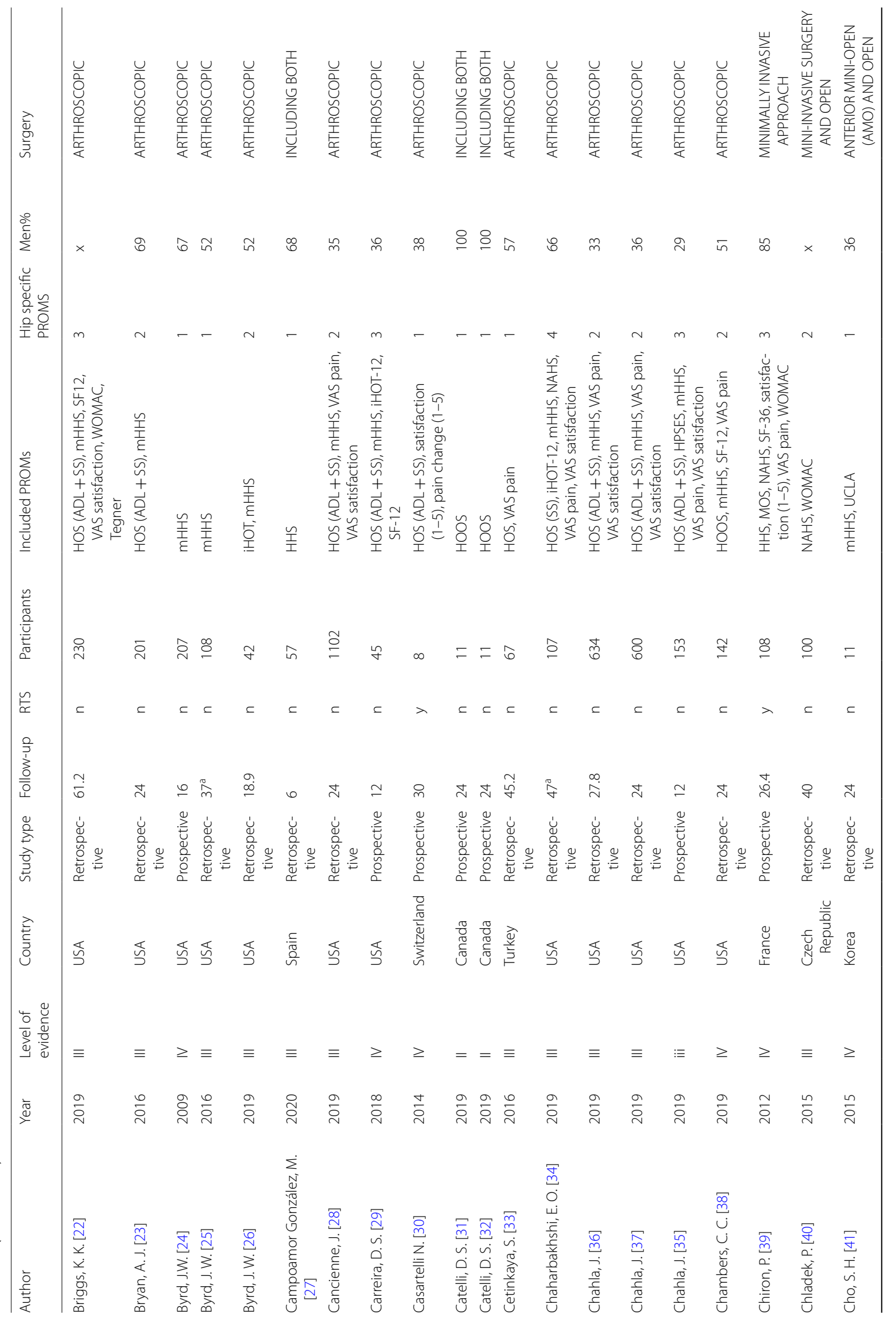


Lindman et al. J EXP ORTOP $\quad$ (2021) 8:33

Page 7 of 26

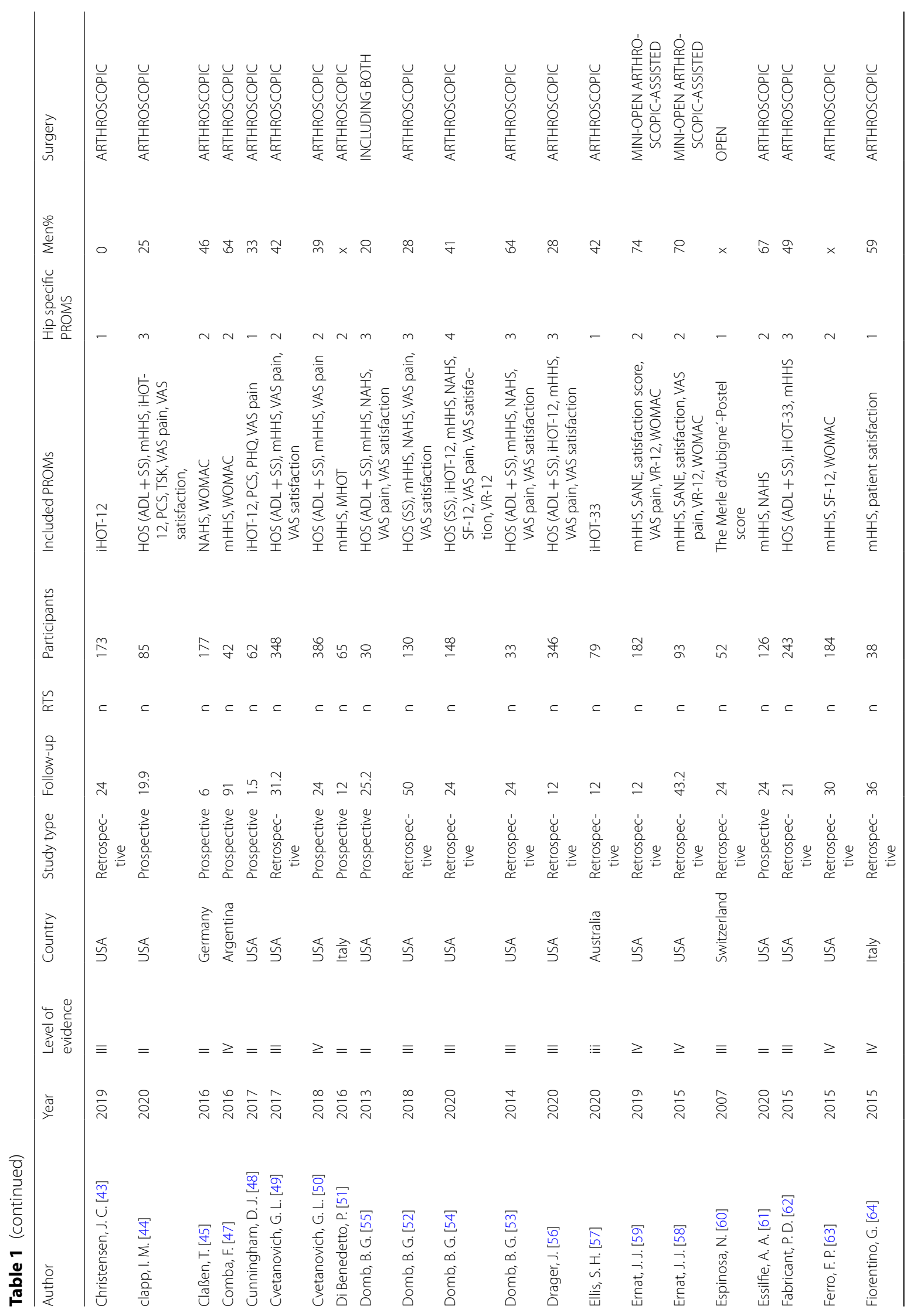




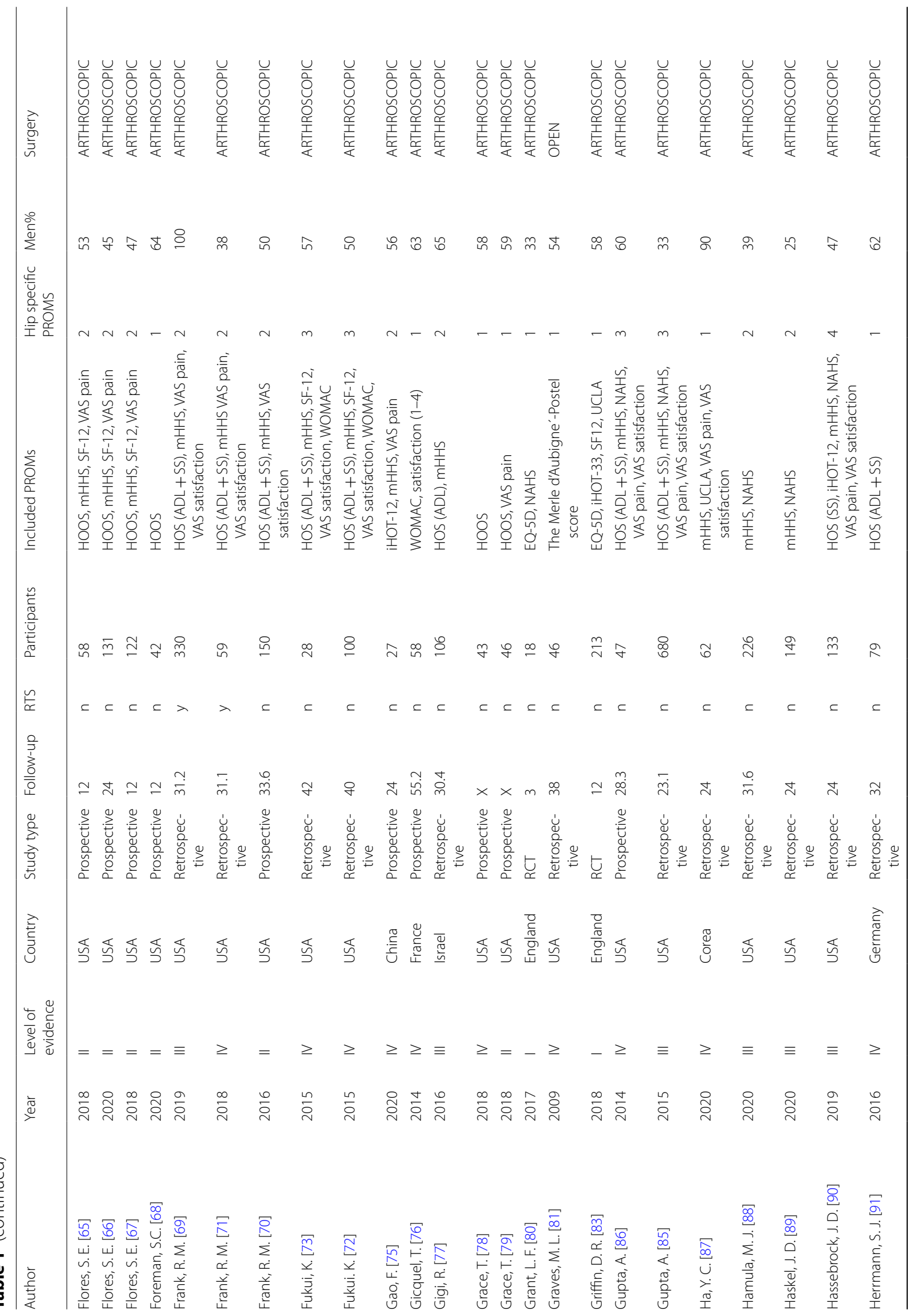




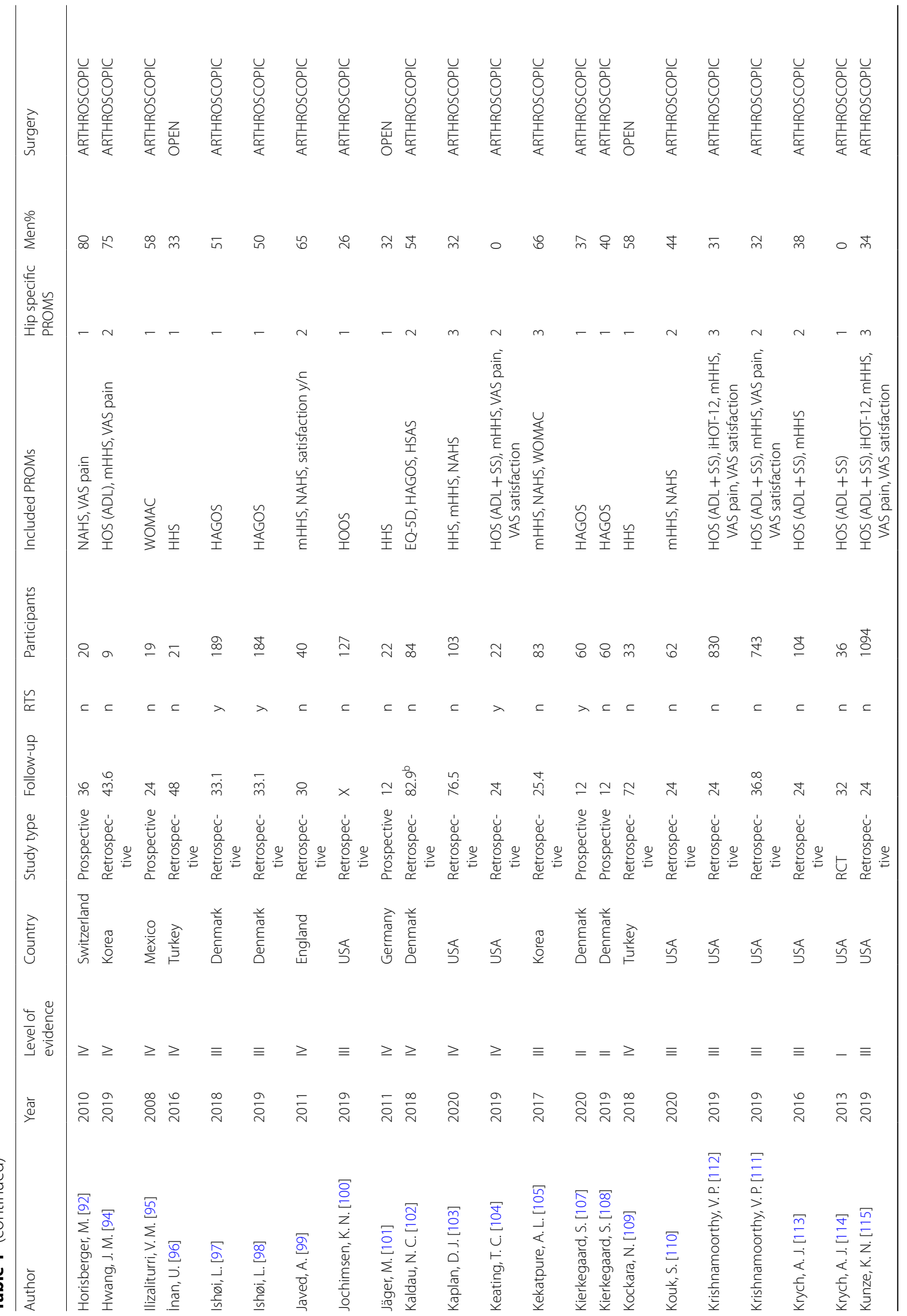




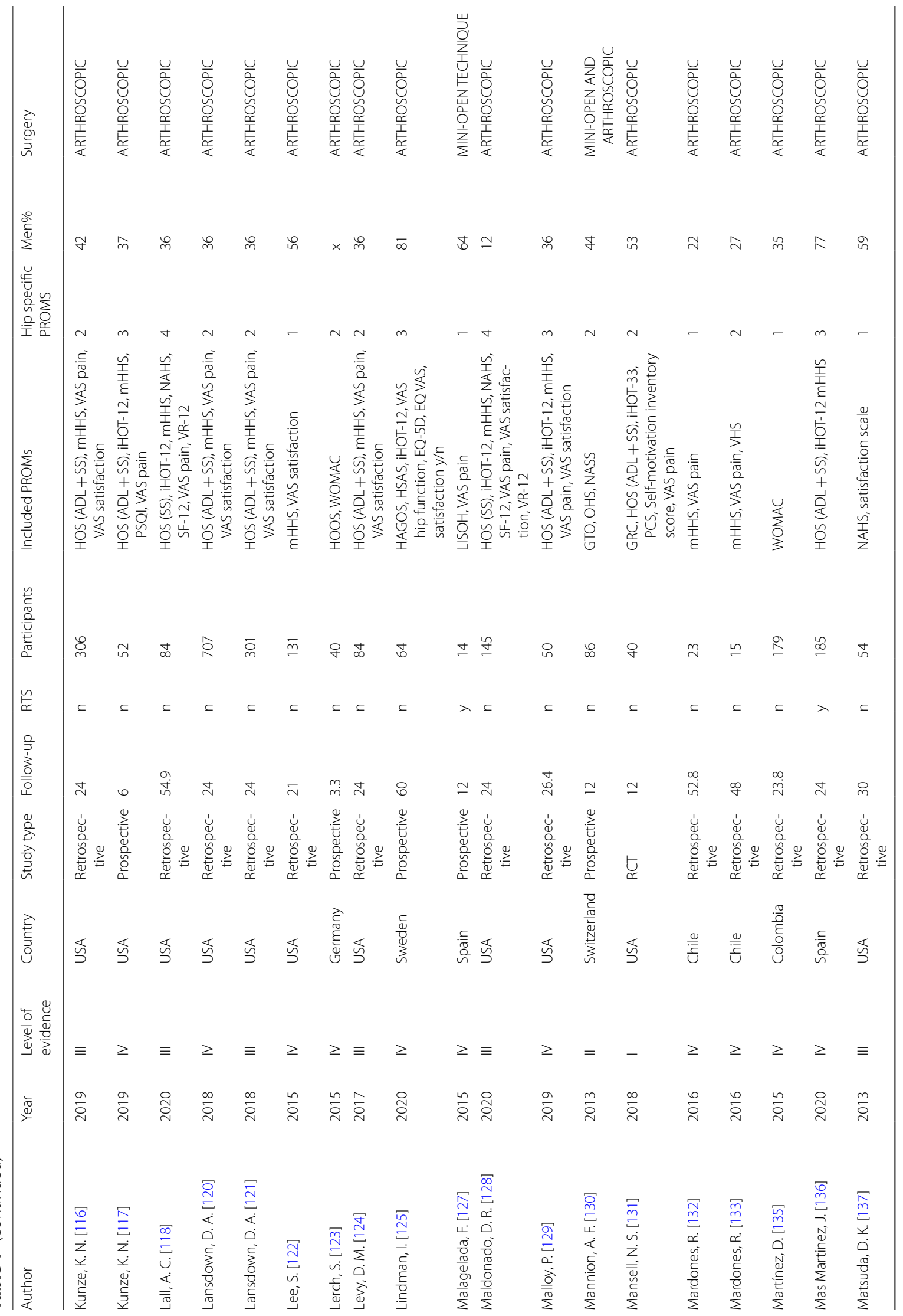




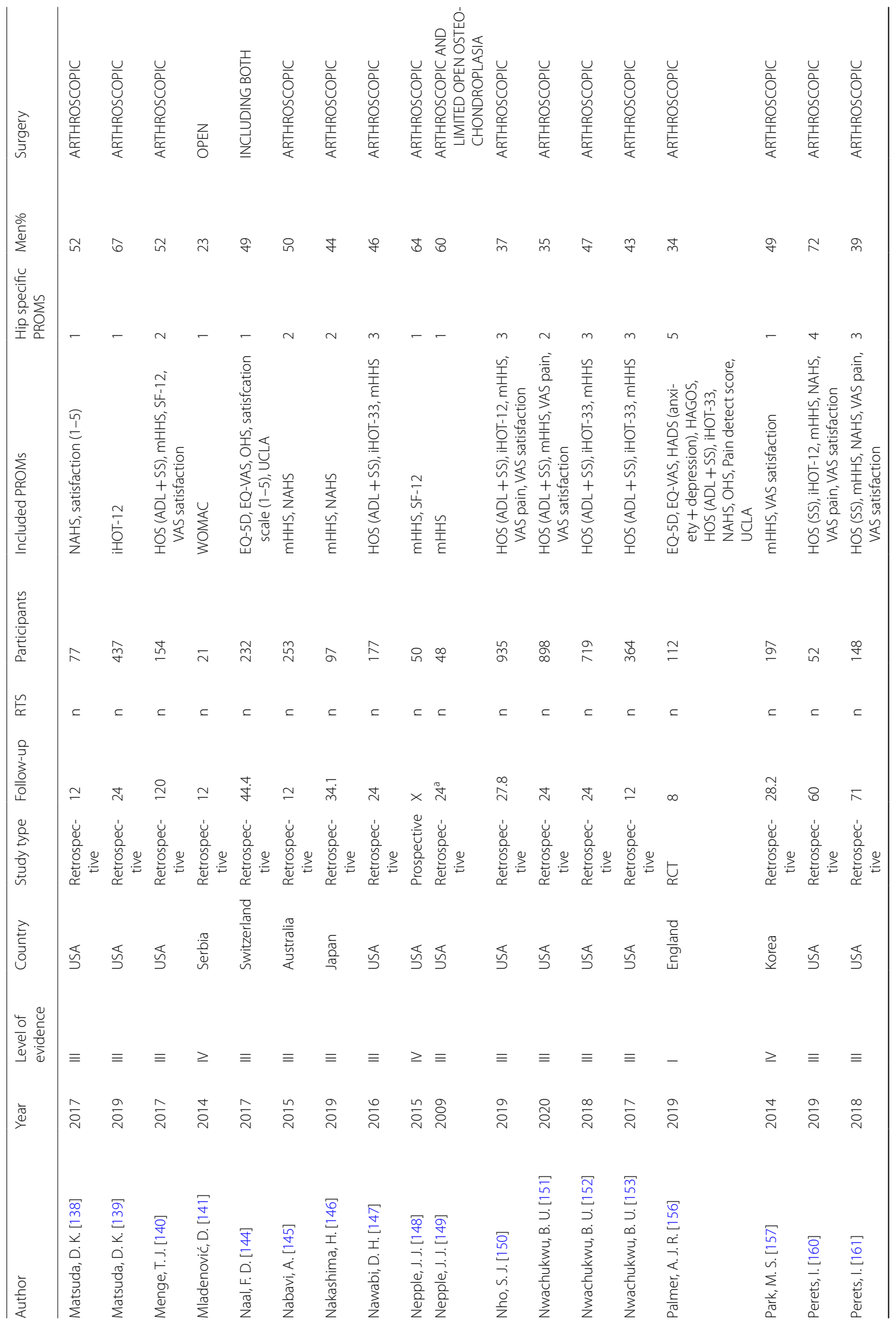




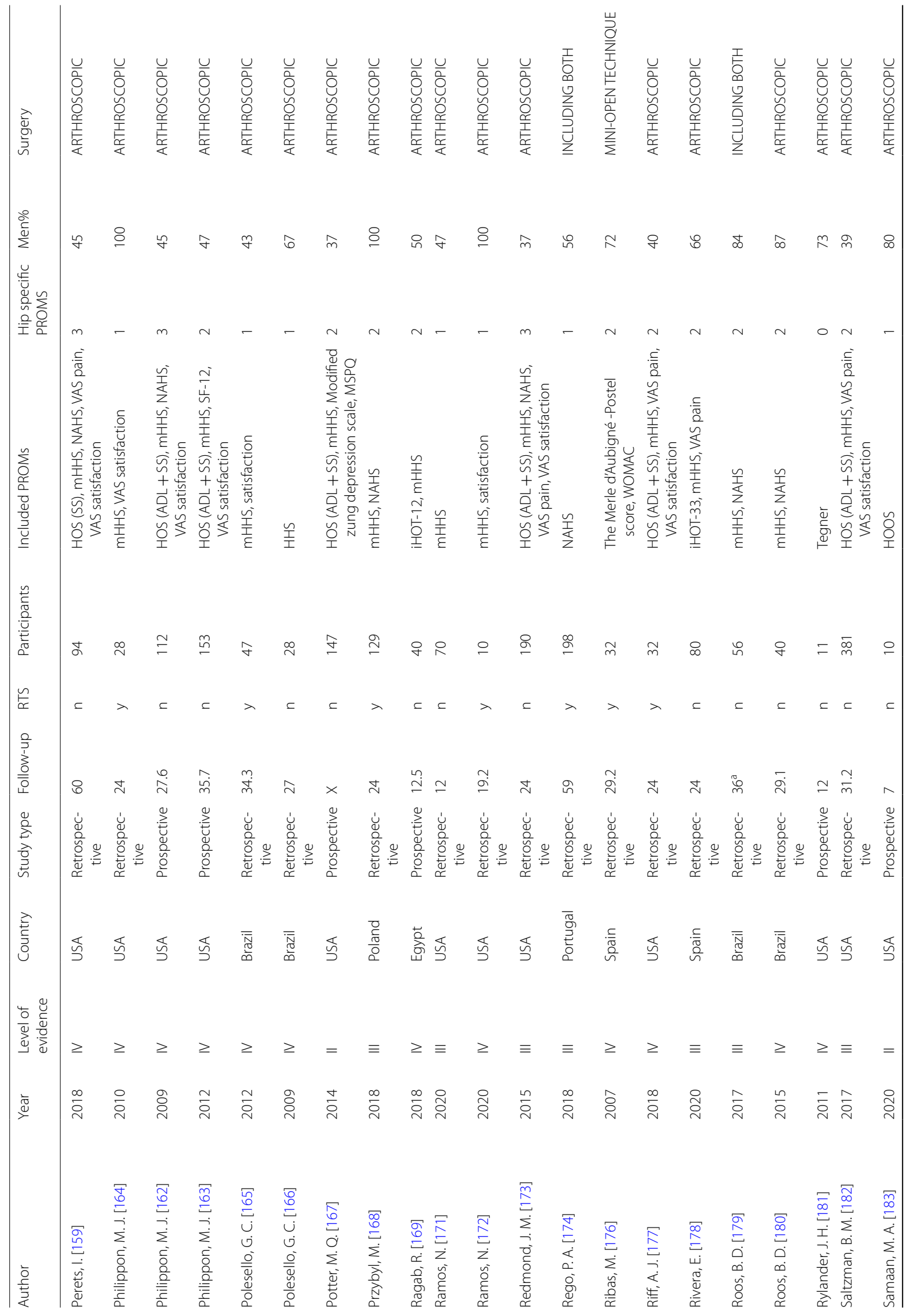




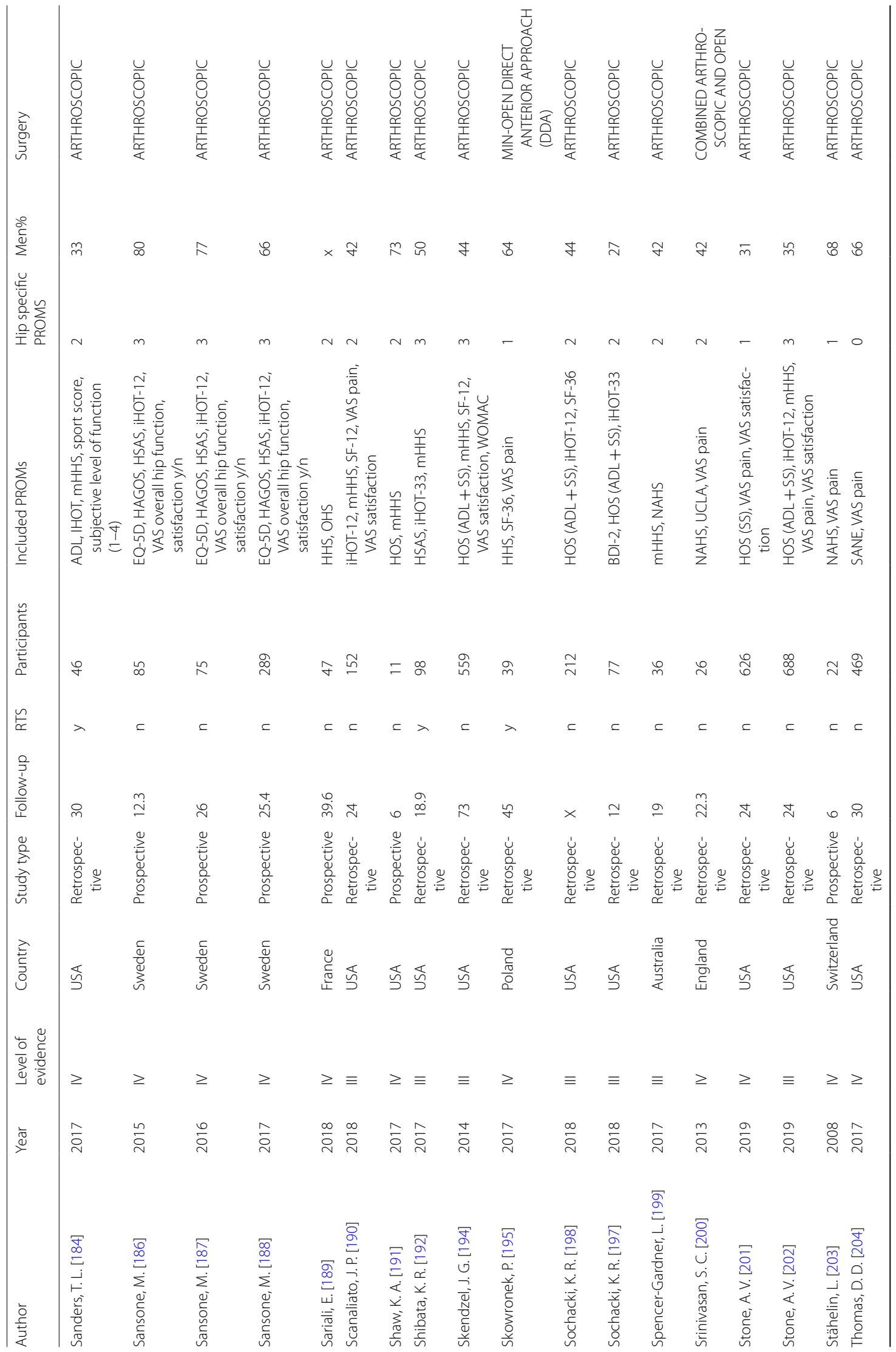




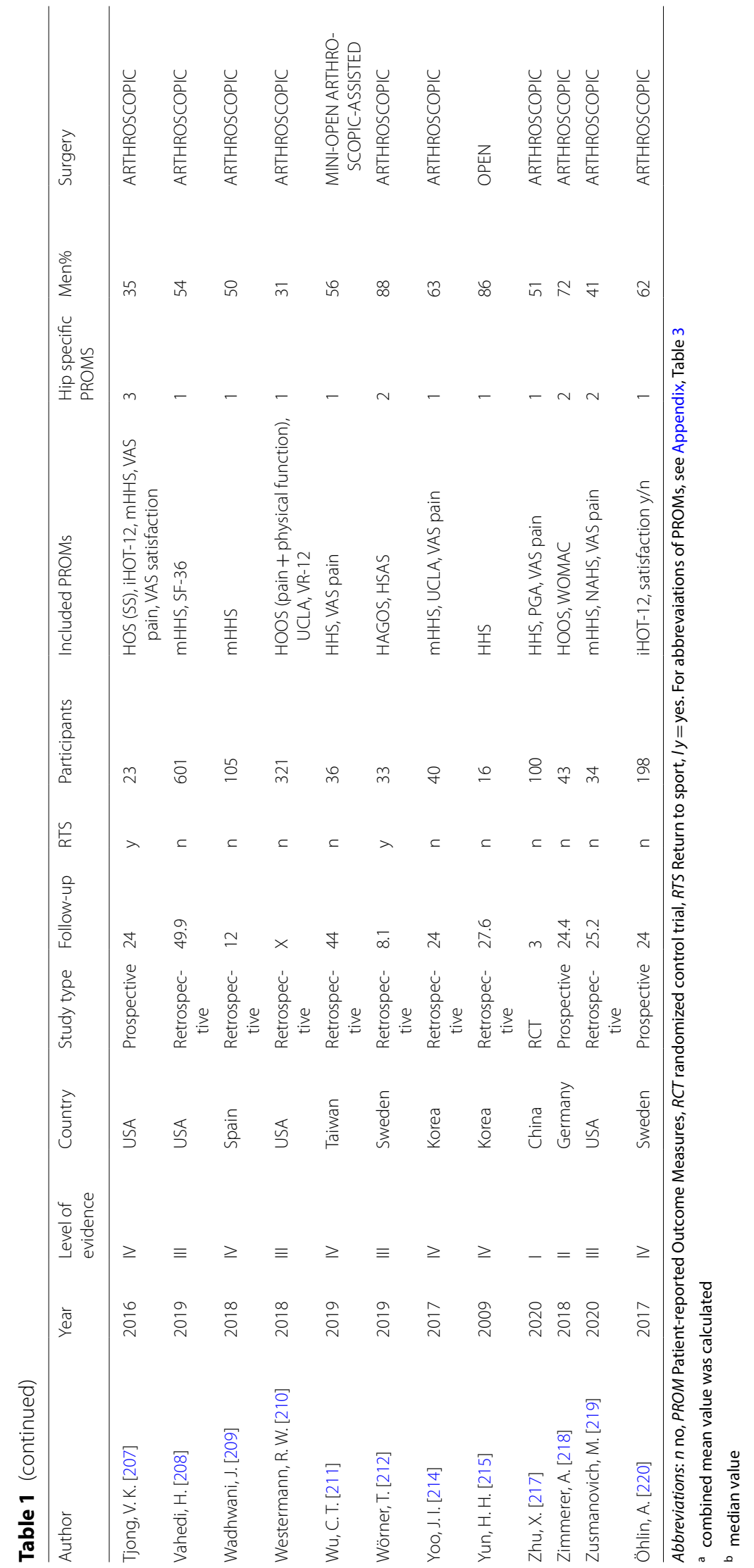




\section{INCLUDED STUDIES}

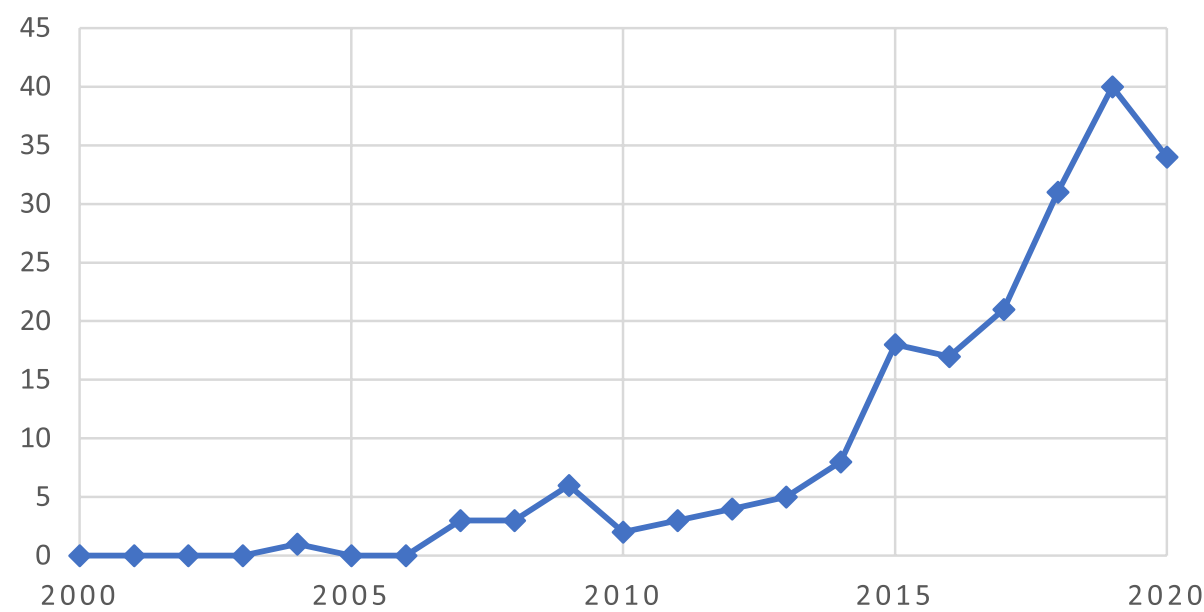

Fig. 2 Trend over the years of included studies. ${ }^{*}$ Note the year 2020 only covers studies until search day 7 th of September

\section{Discussion}

The most important finding in this systematic review was the expected growth in the number of studies published over the years, where over $70 \%$ of the included studies were published between 2016-2020. Although the literature review included studies from 1999-2020, the first study meeting the inclusion criteria was published in 2004.

A total of 39 different PROMs were used among the studies, of which 15 were hip specific. The most common non-hip specific outcome was satisfaction, found in $40 \%$ of the studies. Previous studies have reported that satisfaction is the most frequently used non-hip specific outcome tool, although there is a variability how satisfaction is reported $[175,193]$. The discrepancy in the use of different PROMs has previously been noted and the reason for this is unknown. The routinely use of a specific PROM, the difficulty in changing PROMs once norms have been established and the inevitable retention of the same PROMs to be able to follow a cohort and evaluate long-term outcomes are possible explanations for the divergence in use of PROMs [175].

After the Warwick agreement in 2016, three patientreported outcome measures were considered suitable for the target population of FAIS and were recommended

\section{Trends in PROMs}

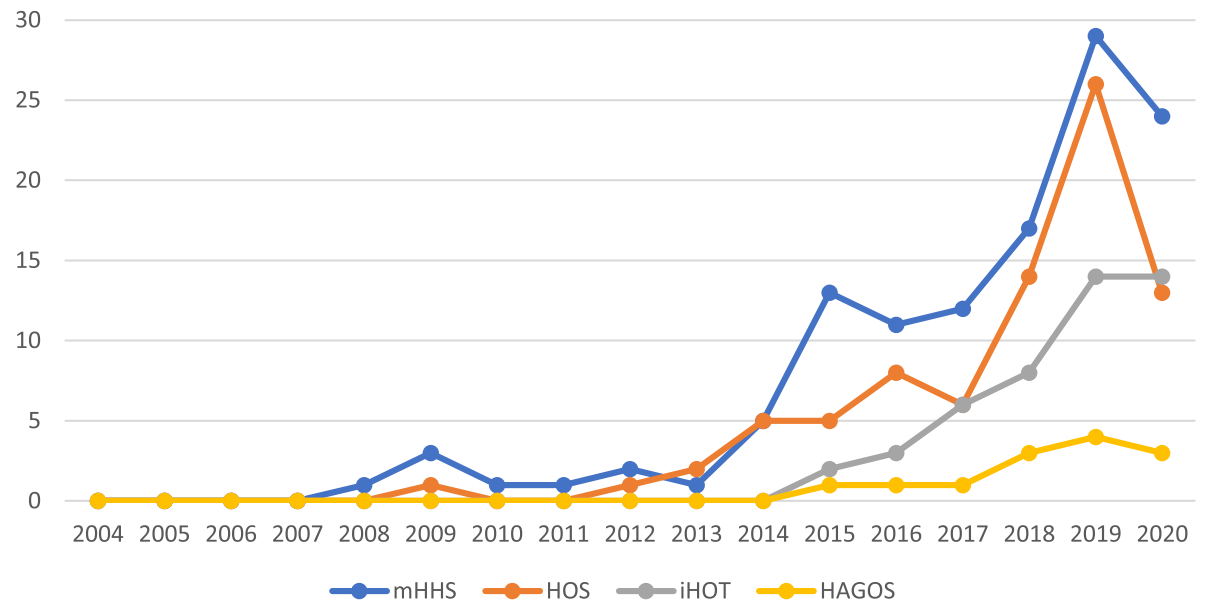

Fig. 3 Trends in the number of recommended PROMs for FAIS and the most commonly used mHHS Abbreviations: HAGOS: The Copenhagen Hip and Groin Outcome Score, HOS: Hip Outcome Score, iHOT: international Hip Outcome Tool, mHHS: modified Harris Hip score, PROM: Patient-reported Outcome Measure. *Note the year 2020 only covers studies until search day $7^{\text {th }}$ of September 
to use when evaluating surgery for FAIS [82], 65\% of the included studies in this systematic review used at least one of the recommended PROMs (HAGOS, iHOT-12 or iHOT-33 and HOS (ADL + SS)). Nonetheless, the mHHS remains being the most commonly used PROM, even though there is a well-known ceiling effect of mHHS described for young active patients [206]. It could be seen as both surprising and concerning that mHHS still is the most used PROM in studies on FAIS as its outcome's validity for young and active patients is considered low. Thorborg et al. [206] found HAGOS to be the best suited PROM for patients with FAIS, which only was used in $7 \%$ of the studies. This finding can guide future healthcare providers and researchers in using hip specific PROMs valid for the target population and diagnosis. Furthermore, there is a need for adoption of new validated scores, translated into the patients' native language.

Only $13 \%$ of the included studies reported RTS specifically by using a clear definition. There is a current challenge in sports science regarding the definition of RTS, and the most optimal evaluation of RTS has not yet been decided. Activity scores such as the HOS (SS), Tegner activity scale or HSAS, with the purpose to evaluate the patients' activity level or issues in sport specific activities, are not the best tools to evaluate the RTS. Mainly because these scores do not include training load or performance compared with preinjury status. This could possibly generate a ceiling effect if the patients rate the PROMs higher, yet still not being capable to fully return to their preinjury level of sport. Furthermore, the definition of RTS has been proposed to differ between elite and recreational athletes [42]. Athletes undergoing hip arthroscopic surgery for FAIS usually have a major interest whether they can RTS again, thus, a reliable method to determine RTS is thus needed.

The majority of the studies were published in USA or in Europe. This has previously been reported [106, 213]. Although USA and Europe have been in the front line of hip arthroscopic surgery and research, a small number of studies included in this systematic review were from Korea and China, indirectly indicating an upcoming trend in performed surgeries for FAIS in Asia. Moreover, only studies in the English language were included in this systematic review, which partly might explain the high percentage of studies from USA and Europe.

Although a few RCT:s have been published, retrospective studies are still the most common. Over the years, patient registries have facilitated prospective evaluation of FAIS and yielded important insight on PROMs [126, 185]. Öhlin et al. [155] assessed the methodological quality of prospective studies over a 5 -year time period and found no improvement in the quality of the methods despite an increase in the number of published studies. With the dramatic increase seen in the number of published studies in this systematic review, it is of importance to also improve the quality of observational studies. New consensus meetings to enhance adoption of suitable PROMs and education of researchers and clinicians could benefit future research in the outcome of FAIS.

\section{Strengths and limitations}

The strength of this study is the methodological rigor using PRISMA guidelines, focus on an important topic and the longitudinal analysis of a 20 -year time horizon.

This systematic review is not without limitations. One of the a-priori set exclusion criteria was age, excluding studies with patients $<18$ years old, though the focus was on the adult population as validation of PROMS in the pediatric population is still emerging. Moreover, only publications in the English language were included and there is a risk of missing publications in non-English speaking countries. Due to the heterogeneity of the included studies no statistical meta-analysis was conducted.

\section{Conclusion}

There has been a continuous increase in the number of published studies regarding FAIS with the majority evaluating arthroscopic surgery. The mHHS remains being the most commonly used PROM. 


\section{Appendix}

Table 2 Search strategy: pubmed $^{a}$

\begin{tabular}{|c|c|c|}
\hline Search & Query & Results \\
\hline \#27 & Search: \#19 NOT \#22 Filters: English Sort by: Most Recent & 2,085 \\
\hline \#23 & Search: \#19 NOT \#22 Sort by: Most Recent & 2,172 \\
\hline \#22 & Search: \#20 OR \#21 Sort by: Most Recent & $5,073,653$ \\
\hline \#21 & $\begin{array}{l}\text { Search: animal[ti] OR animals[ti] OR rat[ti] OR rats[ti] OR mouse[ti] OR mice[ti] OR rodent[ti] } \\
\text { OR rodents[ti] OR dog[ti] OR dogs[ti] OR cat[ti] OR cats[ti] OR koalas[ti] OR hamster[ti] OR } \\
\text { hamsters[ti] OR rabbit[ti] OR rabbits[ti] OR swine[ti] OR murine[ti] Sort by: Most Recent }\end{array}$ & $1,886,518$ \\
\hline \#20 & Search: ((animals[mh]) NOT (animals[mh] AND humans[mh])) Sort by: Most Recent & $4,731,731$ \\
\hline \#19 & Search: \#5 AND \#18 Sort by: Most Recent & 2,177 \\
\hline \#18 & Search: \#6 OR \#7 OR \#17 Sort by: Most Recent & $2,006,557$ \\
\hline$\# 17$ & $\begin{array}{l}\text { Search: surgery[tiab] OR surgical[tiab] OR operative[tiab] OR minimally invasive[tiab] Sort } \\
\text { by: Most Recent }\end{array}$ & $1,989,360$ \\
\hline$\# 7$ & Search: arthroscop*[tiab] Sort by: Most Recent & 31,803 \\
\hline \#6 & Search: "Arthroscopy"[Mesh] Sort by: Most Recent & 23,951 \\
\hline \#5 & Search: \#2 OR \#3 OR \#4 Sort by: Most Recent & 4,313 \\
\hline \#4 & $\begin{array}{l}\text { Search: hip impingement[tiab] OR cam impingement[tiab] OR pincer impingement[tiab] OR } \\
\text { FAI[tiab] OR FAIS[tiab] Sort by: Most Recent }\end{array}$ & 2,865 \\
\hline \#3 & $\begin{array}{l}\text { Search: (femoroacetabular[tiab] OR femoracetabular[tiab] OR femoral acetabular[tiab] OR } \\
\text { femoro-acetabular[tiab]) AND impingement[tiab] Sort by: Most Recent }\end{array}$ & 2,738 \\
\hline \#2 & Search: "Femoracetabular Impingement"[Mesh] Sort by: Most Recent & 1,702 \\
\hline
\end{tabular}

${ }^{a}$ Date of search: 7th of September 2020. Results: 2085 studies 
Table 3 Included patient-reported outcome measures (PROMs) and their abbreviations

\begin{tabular}{|c|c|c|}
\hline PROM & Name & Hip specific \\
\hline $\mathrm{BDI}-2$ & Beck Depression Inventory & No \\
\hline EQ-5D & European Quality of life index version 5D & No \\
\hline FAA & Functional Activity Assessment & No \\
\hline GRC & Global Rating of Change & No \\
\hline GTO & Global Treatment Outcome & No \\
\hline HADS & Hospital Anxiety and Depression Scale & No \\
\hline HAGOS & The Copenhagen Hip and Groin Outcome Score & Yes \\
\hline $\mathrm{HHS}$ & Harris Hip Score & Yes \\
\hline HOOS & Hip Disability and Osteoarthritis Outcome Score & Yes \\
\hline $\mathrm{HOS}(\mathrm{ADL}+\mathrm{SS})$ & Hip Outcome Score (Activities of Daily Living + Sport Specific) & Yes \\
\hline HPSES & Hip Preservation Surgery Expectations Survey & Yes \\
\hline iHOT-12 & The international Hip Outcome Tool-12 & Yes \\
\hline iHOT-33 & The international Hip Outcome Tool-33 & Yes \\
\hline LISHO & Lequesne Functional Index for Hip Osteoarthritis & Yes \\
\hline Merle d'Aubigne and Postel scale & & Yes \\
\hline $\mathrm{mHHS}$ & modified Harris Hip Score & Yes \\
\hline MHOT & Mahorn Hip Outcome Tool & Yes \\
\hline MSPQ & Modified Somatic Perception Questionnaire & No \\
\hline Modified zung depression scale & - & No \\
\hline NASS & North American Spine Society Lumbar Spine Questionnaire & No \\
\hline MOS & Mean Opinion Score & No \\
\hline NAHS & Non-Arthritic Hip Score & Yes \\
\hline $\mathrm{OHS}$ & Oxford Hip Score & Yes \\
\hline Pain detect score & - & No \\
\hline PCS & Pain Catastrophizing Scale & No \\
\hline$P G A$ & Patient Global Assessment & No \\
\hline $\mathrm{PHQ}$ & Patient Health Questionnaire & No \\
\hline PSQI & Pittsburgh Sleep Quality Index & No \\
\hline SANE & Single Assessment Numeric Evaluation & No \\
\hline Satisfaction & & No \\
\hline SF-12 & 12-item Short-Form Health Survey & No \\
\hline SF-36 & The Short Form 36 Health Survey & No \\
\hline Tegner & - & No \\
\hline TSK & Tampa Scale of Kinesiophobia & No \\
\hline UCLA & University of California Los Angeles activity scores. & No \\
\hline VAS pain & Visual analoge scale & No \\
\hline VHS & Vail Hip score & Yes \\
\hline VR-12 & The Veterans RAND 12 Item Health Survey & No \\
\hline WOMAC & Western Ontario and MacMaster Universities Osteoarthritis Index & Yes \\
\hline
\end{tabular}




\begin{abstract}
Abbreviations
FAIS: Femoroacetabular impingement syndrome; HAGOS: Hip and Groin outcome score; iHOT: International Hip Outcome Tool; mHHS: Modified Harris Hip Score; PRISMA: Preferred Reporting Items for Systematic Review and Meta-Analysis; PROM: Patient-reported outcome measure; RCT: Randmoized controlled trial; RTS: Return to sports; SD: Standard Deviation; WOMAC: Western Ontario and McMaster Universities Osteoarthritis Index.
\end{abstract}

\section{Acknowledgments}

Not applicable.

\section{Authors' contributions}

IL: Study idea, literature screening, data collection, data analysis, manuscript writing. SN: Literature screening, manuscript writing. AÖ: Study idea, manuscript writing. EHS: Manuscript writing. OA: Study idea, manuscript writing. JK: Manuscript writing. MS: Study idea, manuscript writing. All authors read and approved the final manuscript.

\section{Funding}

Open access funding provided by University of Gothenburg.

\section{Availability of data and materials}

All data analyzed is included in the published study and its supplementary information files or references.

\section{Declarations}

\section{Ethics approval and consent to participate}

Not applicable.

\section{Consent for publication}

Not applicable.

\section{Competing interests}

OA declare a potential conflict of interest as a non-financial arrangement of "Speakers Bureau of Conmed". Other authors have no competing interest to declare.

\section{Author details}

1 Department of Orthopaedics, Institute of Clinical Sciences, Sahlgrenska Academy, University of Gothenburg, 41345 Gothenburg, Sweden. ${ }^{2}$ Department of Orthopaedic Surgery, South Älvsborg Hospital, 50182 Borås, Sweden. ${ }^{3}$ Department of Health and Rehabilitation, Institute of Neuroscience and Physiology, Sahlgrenska Academy, University of Gothenburg, Gothenburg, Sweden. ${ }^{4}$ Division of Orthopaedic Surgery, McMaster University, Hamilton, ON L8N 3Z5, Canada.

\section{Received: 5 February 2021 Accepted: 8 April 2021}

Published online: 23 April 2021

\section{References}

1. Abrahamson J, Lindman I, Sansone M, Öhlin A, Jonasson P, Karlsson J, Baranto A (2020) Low rate of high-level athletes maintained a return to pre-injury sports two years after arthroscopic treatment for femoroacetabular impingement syndrome. J Exp Orthop 7(1):44. https://doi.org/ 10.1186/s40634-020-00263-5

2. Aguilera-Bohórquez B, Ramirez S, Cantor E (2020) Functional results of arthroscopic treatment in patients with femoroacetabular and subspine impingement diagnosed with a 3-dimensional dynamic study. Arthrosc Sports Med Rehabil 2(1):e39-e45. https://doi.org/10.1016/j. asmr.2019.10.007

3. Atzmon R, Sharfman ZT, Haviv B, Frankl M, Rotem G, Amar E, Drexler M, Rath E (2019) Does capsular closure influence patient-reported outcomes in hip arthroscopy for femoroacetabular impingement and labral tear? J Hip Preserv Surg 6(3):199-206. https://doi.org/10.1093/ jhps/hnz025
4. Avnieli IB, Vidra M, Factor S, Atzmon R, Persitz J, Safran N, Rath E, Amar E (2020) Postoperative weightbearing protocols after arthroscopic surgery for femoroacetabular impingement does not affect patient outcome: a comparative study with minimum 2-year follow-up. Arthroscopy 36(1):159-164. https://doi.org/10.1016/j.arthro.2019.08.012

5. Balazs GC, Donohue MA, Brelin AM, Brooks DI, McCabe MP, Anderson TD (2018) Reaction time and brake pedal depression following arthroscopic hip surgery: a prospective case-control study. Arthroscopy 34(5):1463-1470.e1461. https://doi.org/10.1016/j.arthro.2018.02.030

6. Barastegui D, Seijas R, Alvarez-Diaz P, Rivera E, Alentorn-Geli E, Steinbacher G, Cuscó X, Cugat R (2018) Assessing long-term return to play after hip arthroscopy in football players evaluating risk factors for good prognosis. Knee Surg Sports Traumatol Arthrosc 26(3):963-968. https://doi.org/10.1007/s00167-017-4573-z

7. Bardakos NV, Vasconcelos JC, Villar RN (2008) Early outcome of hip arthroscopy for femoroacetabular impingement: the role of femoral osteoplasty in symptomatic improvement. J Bone Joint Surg $\mathrm{Br}$ 90(12):1570-1575. https://doi.org/10.1302/0301-620x.90b12.21012

8. Basques BA, Waterman BR, Ukwuani G, Beck EC, Neal WH, Friel NA, Stone AV, Nho SJ (2019) Preoperative symptom duration is associated with outcomes after hip arthroscopy. Am J Sports Med 47(1):131137. https://doi.org/10.1177/0363546518808046

9. Beaulé PE, Le Duff MJ, Zaragoza E (2007) Quality of life following femoral head-neck osteochondroplasty for femoroacetabular impingement. J Bone Joint Surg Am 89(4):773-779. https://doi.org/ 10.2106/jbjs.f.00681

10. Beaulé PE, Speirs AD, Anwander H, Melkus G, Rakhra K, Frei H, Lamontagne M (2017) Surgical correction of cam deformity in association with femoroacetabular impingement and its impact on the degenerative process within the hip joint. J Bone Joint Surg Am 99(16):1373-1381. https://doi.org/10.2106/jbjs.16.00415

11. Beck EC, Drager J, Nwachukwu BU, Rasio J, Jan K, Chahla J, Nho SJ (2020) Patients with borderline hip dysplasia achieve clinically signifcant improvement after arthroscopic femoroacetabular impingement surgery: a case-control study with a minimum 5-year follow-up. Am J Sports Med 48(7):1616-1624. https://doi.org/10.1177/03635 46520916473

12. Beck EC, Kunze KN, Friel NA, Neal WH, Fu MC, Giordano BD, Chahla J, Nho SJ (2019) Is there a correlation between outcomes after hip arthroscopy for femoroacetabular impingement syndrome and patient cortical bone thickness? J Hip Preserv Surg 6(1):16-24. https://doi.org/10.1093/jhps/hnz010

13. Beck EC, Nwachukwu BU, Chahla J, Jan K, Keating TC, Suppauksorn S, Nho SJ (2019) Patients with borderline hip dysplasia achieve clinically significant outcome after arthroscopic femoroacetabular impingement surgery: a case-control study with minimum 2-year follow-up. Am J Sports Med 47(11):2636-2645. https://doi.org/10.1177/03635 46519865919

14. Beck EC, Nwachukwu BU, Chapman R, Gowd AK, Waterman BR, Nho SJ (2020) The influence of lumbosacral spine pathology on minimum 2-year outcome after hip arthroscopy: a nested case-control analysis. Am J Sports Med 48(2):403-408. https://doi.org/10.1177/0363546519 892916

15. Beck EC, Nwachukwu BU, Jan K, Krivicich LM, Chahla J, Fu MC, Nho SJ (2020) The effect of postoperative opioid prescription refills on achieving meaningful clinical outcomes after hip arthroscopy for femoroacetabular impingement syndrome. Arthroscopy 36(6):1599-1607. https:// doi.org/10.1016/j.arthro.2020.02.007

16. Beck EC, Nwachukwu BU, Krivicich LM, Malloy P, Suppauksorn S, Jan K, Nho SJ (2020) Preoperative hip extension strength is an independent predictor of achieving clinically significant outcomes after hip arthroscopy for femoroacetabular impingement syndrome. Sports Health 12(4):361-372. https://doi.org/10.1177/1941738120910134

17. Beck EC, Nwachukwu BU, Lee EK, Chapman R, Stubbs AJ, Gitelis M, Rasio J, Nho SJ (2020) Travel distance does not affect outcomes in hip preservation surgery: a case for centers of excellence. Orthop J Sports Med 8(3):2325967120908821. https://doi.org/10.1177/2325967120 908821

18. Beck M, Leunig M, Parvizi J, Boutier V, Wyss D, Ganz R (2004) Anterior femoroacetabular impingement: part II. Midterm results of surgical treatment. Clin Orthop Relat Res 418:67-73 
19. Bennett AN, Nixon J, Roberts A, Barker-Davies R, Villar R, Houghton JM (2016) Prospective 12-month functional and vocational outcomes of hip arthroscopy for femoroacetabular impingement as part of an evidence-based hip pain rehabilitation pathway in an active military population. BMJ Open Sport Exerc Med 2(1):e000144. https://doi.org/ 10.1136/bmjsem-2016-000144

20. Bolia IK, Fagotti L, Briggs KK, Philippon MJ (2019) Midterm outcomes following repair of capsulotomy versus nonrepair in patients undergoing hip arthroscopy for femoroacetabular impingement with labral repair. Arthroscopy 35(6):1828-1834. https://doi.org/10.1016/j.arthro. 2019.01.033

21. Boone GR, Pagnotto MR, Walker JA, Trousdale RT, Sierra RJ (2012) Caution should be taken in performing surgical hip dislocation for the treatment of femoroacetabular impingement in patients over the age of 40. HSS J 8(3):230-234. https://doi.org/10.1007/s11420-012-9306-8

22. Briggs KK, Soares E, Bhatia S, Philippon MJ (2019) Postoperative alpha angle not associated with patient-centered midterm outcomes following hip arthroscopy for FAl. Knee Surg Sports Traumatol Arthrosc 27(10):3105-3109. https://doi.org/10.1007/s00167-018-4933-3

23. Bryan AJ, Krych AJ, Pareek A, Reardon PJ, Berardelli R, Levy BA (2016) Are short-term outcomes of hip arthroscopy in patients 55 years and older inferior to those in younger patients? Am J Sports Med 44(10):25262530. https://doi.org/10.1177/0363546516652114

24. Byrd JW, Jones KS (2009) Arthroscopic femoroplasty in the management of cam-type femoroacetabular impingement. Clin Orthop Relat Res 467(3):739-746. https://doi.org/10.1007/s11999-008-0659-8

25. Byrd JW, Jones KS, Freeman CR (2016) Surgical outcome of pincer femoroacetabular impingement with and without labral ossification. Arthroscopy 32(6):1022-1029. https://doi.org/10.1016/j.arthro.2015.12. 042

26. Byrd JWT, Jones KS (2019) Arthroscopic acetabular labral repair in patients over the age of 60 years: a matched case-control study. Arthroscopy 35(5):1406-1410. https://doi.org/10.1016/j.arthro.2018.11. 015

27. Campoamor González M, Martínez Aznar C, Martín Martínez A, Martín Hernández C, Mateo Agudo JJ, Panisello Sebastiá JJ (2020) Comparison of the open versus the arthroscopic approach in the treatment of femoroacetabular shock. Rev Esp Cir Ortop Traumatol 64(3):185-190. https://doi.org/10.1016/j.recot.2019.11.006

28. Cancienne J, Kunze KN, Beck EC, Chahla J, Suppauksorn S, Nho SJ (2019) Influence of cigarette smoking at the time of surgery on postoperative outcomes in patients with femoroacetabular impingement: a matchedpair cohort analysis. Am J Sports Med 47(5):1138-1144. https://doi.org/ $10.1177 / 0363546519832545$

29. Carreira DS, Kruchten MC, Emmons BR, Startzman AN, Martin RL (2018) A characterization of sensory and motor neural dysfunction in patients undergoing hip arthroscopic surgery: traction- and portal placementrelated nerve injuries. Orthop J Sports Med 6(9):2325967118797306. https://doi.org/10.1177/2325967118797306

30. Casartelli NC, Maffiuletti NA, Item-Glatthorn JF, Impellizzeri FM, Leunig M (2014) Hip muscle strength recovery after hip arthroscopy in a series of patients with symptomatic femoroacetabular impingement. Hip Int 24(4):387-393. https://doi.org/10.5301/hipint.5000131

31. Catelli DS, Kowalski E, Beaulé PE, Lamontagne M (2019) Increased pelvic mobility and altered hip muscles contraction patterns: two-year follow-up cam-FAIS corrective surgery. J Hip Preserv Surg 6(2):140-148. https://doi.org/10.1093/jhps/hnz019

32. Catelli DS, Ng KCG, Kowalski E, Beaulé PE, Lamontagne M (2019) Modified gait patterns due to cam FAl syndrome remain unchanged after surgery. Gait Posture 72:135-141. https://doi.org/10.1016/j.gaitpost. 2019.06.003

33. Cetinkaya S, Toker B, Ozden VE, Dikmen G, Taser O (2016) Arthroscopic labral repair versus labral debridement in patients with femoroacetabular impingement: a minimum 2.5 year follow-up study. Hip Int 26(1):20-24. https://doi.org/10.5301/hipint.5000290

34. Chaharbakhshi EO, Hartigan DE, Spencer JD, Perets I, Lall AC, Domb BG (2019) Do larger acetabular chondral defects portend inferior outcomes in patients undergoing arthroscopic acetabular microfracture? A matched-controlled study. Arthroscopy 35(7):2037-2047. https://doi. org/10.1016/j.arthro.2019.01.047
35. Chahla J, Beck EC, Nwachukwu BU, Alter T, Harris JD, Nho SJ (2019) Is there an association between preoperative expectations and patientreported outcome after hip arthroscopy for femoroacetabular impingement syndrome? Arthroscopy 35(12):3250-3258.e3251. https://doi.org/ 10.1016/j.arthro.2019.06.018

36. Chahla J, Beck EC, Okoroha K, Cancienne JM, Kunze KN, Nho SJ (2019) Prevalence and clinical implications of chondral injuries after hip arthroscopic surgery for femoroacetabular impingement syndrome. Am J Sports Med 47(11):2626-2635. https://doi.org/10.1177/0363546519 865912

37. Chahla J, Nwachukwu BU, Beck EC, Neal WH, Cancienne J, Okoroha KR, Ahn J, Nho SJ (2019) Influence of acetabular labral tear length on outcomes after hip arthroscopy for femoroacetabular impingement syndrome with capsular plication. Am J Sports Med 47(5):1145-1150. https://doi.org/10.1177/0363546519831291

38. Chambers CC, Monroe EJ, Flores SE, Borak KR, Zhang AL (2019) Periportal capsulotomy: technique and outcomes for a limited capsulotomy during hip arthroscopy. Arthroscopy 35(4):1120-1127. https://doi.org/ 10.1016/j.arthro.2018.10.142

39. Chiron P, Espié A, Reina N, Cavaignac E, Molinier F, Laffosse JM (2012) Surgery for femoroacetabular impingement using a minimally invasive anterolateral approach: analysis of 118 cases at 2.2-year follow-up. Orthop Traumatol Surg Res 98(1):30-38. https://doi.org/10.1016/j.otsr. 2011.08.011

40. Chladek P, Musalek M, Trc T, Zahradnik P, Kos P (2015) Femoroacetabular impingement syndrome-efficacy of surgical treatment with regards to age and basic diagnosis. Int Orthop 39(3):417-422. https://doi.org/10. 1007/s00264-014-2574-9

41. Cho SH (2015) Open surgical treatment for femoroacetabular impingement in patients over thirty years: two years follow-up results. Hip Pelvis 27(4):241-249. https://doi.org/10.5371/hp.2015.27.4.241

42. Chona DV, Bonano JC, Ayeni OR, Safran MR (2020) Definitions of return to sport after hip arthroscopy: are we speaking the same language and are we measuring the right outcome? Orthop J Sports Med 8(9):2325967120952990. https://doi.org/10.1177/2325967120952990

43. Christensen JC, Marland JD, Miller CJ, Horton BS, Whiting DR, West HS (2019) Trajectory of clinical outcomes following hip arthroscopy in female subgroup populations. J Hip Preserv Surg 6(1):25-32. https:// doi.org/10.1093/jhps/hnz011

44. Clapp IM, Nwachukwu BU, Beck EC, Rasio JP, Alter T, Allison B, Nho SJ (2020) What is the role of kinesiophobia and pain catastrophizing in outcomes after hip arthroscopy for femoroacetabular impingement syndrome? Arthrosc Sports Med Rehabil 2(2):e97-e104. https://doi.org/ 10.1016/j.asmr.2019.12.001

45. Claßen T, Körsmeier K, Kamminga M, Beck S, Rekowski J, Jäger M, Landgraeber S (2016) Is early treatment of cam-type femoroacetabular impingement the key to avoiding associated full thickness isolated chondral defects? Knee Surg Sports Traumatol Arthrosc 24(7):23322337. https://doi.org/10.1007/s00167-014-3332-7

46. Colvin AC, Harrast J, Harner C (2012) Trends in hip arthroscopy. J Bone Joint Surg Am 94(4):e23. https://doi.org/10.2106/JBJS.J.01886

47. Comba F, Yacuzzi C, Ali PJ, Zanotti G, Buttaro M, Piccaluga F (2016) Joint preservation after hip arthroscopy in patients with FAI. Prospective analysis with a minimum follow-up of seven years. Muscles Ligaments Tendons J 6(3):317-323. https://doi.org/10.11138/m/tj/2016.6.3.317

48. Cunningham DJ, Lewis BD, Hutyra CA, Mather RC, Olson SA (2017) Early recovery after hip arthroscopy for femoroacetabular impingement syndrome: a prospective, observational study. J Hip Preserv Surg 4(4):299-307. https://doi.org/10.1093/jhps/hnx026

49. Cvetanovich GL, Levy DM, Weber AE, Kuhns BD, Mather RC 3rd, Salata MJ, Nho SJ (2017) Do patients with borderline dysplasia have inferior outcomes after hip arthroscopic surgery for femoroacetabular impingement compared with patients with normal acetabular coverage? Am J Sports Med 45(9):2116-2124. https://doi.org/10.1177/0363546517 702855

50. Cvetanovich GL, Weber AE, Kuhns BD, Alter J, Harris JD, Mather RC 3rd, Nho SJ (2018) Hip arthroscopic surgery for femoroacetabular impingement with capsular management: factors associated with achieving clinically significant outcomes. Am J Sports Med 46(2):288-296. https:// doi.org/10.1177/0363546517739824 
51. Di Benedetto P, Barbattini P, Povegliano L, Beltrame A, Gisonni R, Cainero V, Causero A (2016) Extracapsular vs standard approach in hip arthroscopy. Acta Biomed 87(Suppl 1):41-45

52. Domb BG, Chaharbakhshi EO, Perets I, Walsh JP, Yuen LC, Ashberg LJ (2018) Patient-reported outcomes of capsular repair versus capsulotomy in patients undergoing hip arthroscopy: minimum 5-year follow-up-a matched comparison study. Arthroscopy 34(3):853-863. e851. https://doi.org/10.1016/j.arthro.2017.10.019

53. Domb BG, El Bitar YF, Stake CE, Trenga AP, Jackson TJ, Lindner D (2014) Arthroscopic labral reconstruction is superior to segmental resection for irreparable labral tears in the hip: a matched-pair controlled study with minimum 2-year follow-up. Am J Sports Med 42(1):122-130. https://doi.org/10.1177/0363546513508256

54. Domb BG, Kyin C, Rosinsky PJ, Shapira J, Yelton MJ, Meghpara MB, Lall AC, Maldonado DR (2020) Circumferential labral reconstruction for irreparable labral tears in the primary setting: minimum 2-year outcomes with a nested matched-pair labral repair control group. Arthroscopy. https://doi.org/10.1016/j.arthro.2020.02.014

55. Domb BG, Stake CE, Botser IB, Jackson TJ (2013) Surgical dislocation of the hip versus arthroscopic treatment of femoroacetabular impingement: a prospective matched-pair study with average 2-year follow-up. Arthroscopy 29(9):1506-1513. https://doi.org/10.1016/j.arthro.2013.06. 010

56. Drager J, Rasio J, Newhouse A, Beck E, Chahla J, Nho SJ (2020) Patients with a hypotrophic labrum achieve similar outcomes after primary labral repair compared with patients with a normal-sized labrum: a matched cohort analysis of 346 patients with femoroacetabular impingement syndrome. Arthroscopy. https://doi.org/10.1016/j.arthro. 2020.05.039

57. Ellis SH, Perriman DM, Burns AWR, Neeman TM, Lynch JT, Smith PN (2020) Total volume of cam deformity alone predicts outcome in arthroscopy for femoroacetabular impingement. Knee Surg Sports Traumatol Arthrosc 28(4):1283-1289. https://doi.org/10.1007/ s00167-019-05383-9

58. Ernat JJ, Song DJ, Brugman SC, Shaha SH, Tokish JM, Lee GY (2015) Mental health medication use correlates with poor outcome after femoroacetabular impingement surgery in a military population. J Bone Joint Surg Am 97(15):1272-1277. https://doi.org/10.2106/jbjs.o. 00043

59. Ernat JJ, Song DJ, Cage JM, Lee GY, Tokish JM (2019) Return to duty after mini-open arthroscopic-assisted treatment of femoroacetabular impingement in an active military population. Arthrosc Sports Med Rehabil 1(1):e15-e23. https://doi.org/10.1016/j.asmr.2019.07.003

60. Espinosa N, Beck M, Rothenfluh DA, Ganz R, Leunig M (2007) Treatment of femoro-acetabular impingement: preliminary results of labral refixation. Surgical technique. J Bone Joint Surg Am 89(Suppl 2 Pt.1):36-53. https://doi.org/10.2106/jbjs.f.01123

61. Essilfie AA, Bloom DA, Zusmanovich M, Kester B, Wolfson T, Youm T (2020) Staged bilateral hip arthroscopy compared with a matched unilateral hip arthroscopy group: minimum 2-year follow-up. Arthroscopy 36(7):1856-1861. https://doi.org/10.1016/j.arthro.2020.02.025

62. Fabricant PD, Fields KG, Taylor SA, Magennis E, Bedi A, Kelly BT (2015) The effect of femoral and acetabular version on clinical outcomes after arthroscopic femoroacetabular impingement surgery. J Bone Joint Surg Am 97(7):537-543. https://doi.org/10.2106/jbjs.n.00266

63. Ferro FP, Ho CP, Briggs KK, Philippon MJ (2015) Patient-centered outcomes after hip arthroscopy for femoroacetabular impingement and labral tears are not different in patients with normal, high, or low femoral version. Arthroscopy 31(3):454-459. https://doi.org/10.1016/j. arthro.2014.10.008

64. Fiorentino G, Fontanarosa A, Cepparulo R, Guardoli A, Berni L, Coviello G, Guardoli A (2015) Treatment of cam-type femoroacetabular impingement. Joints 3(2):67-71. https://doi.org/10.11138/jts/2015.3.2.067

65. Flores SE, Borak KR, Zhang AL (2018) Hip arthroscopic surgery for femoroacetabular impingement: a prospective analysis of the relationship between surgeon experience and patient outcomes. Orthop J Sports Med 6(2):2325967118755048. https://doi.org/10.1177/2325967118 755048

66. Flores SE, Chambers CC, Borak KR, Zhang AL (2020) Is there a gender gap in outcomes after hip arthroscopy for femoroacetabular impingement? Assessment of clinically meaningful improvements in a prospective cohort. Orthop J Sports Med 8(7):2325967119900561. https://doi.org/10.1177/2325967119900561

67. Flores SE, Sheridan JR, Borak KR, Zhang AL (2018) When do patients improve after hip arthroscopy for femoroacetabular impingement? A prospective cohort analysis. Am J Sports Med 46(13):3111-3118. https://doi.org/10.1177/0363546518795696

68. Foreman SC, Zhang AL, Neumann J, von Schacky CE, Souza RB, Majumdar S, Link TM (2020) Postoperative MRI findings and associated pain changes after arthroscopic surgery for femoroacetabular impingement. AJR Am J Roentgenol 214(1):177-184. https://doi.org/10.2214/ajr.19. 21421

69. Frank RM, Kunze KN, Beck EC, Neal WH, Bush-Joseph CA, Nho SJ (2019) Do female athletes return to sports after hip preservation surgery for femoroacetabular impingement syndrome?: A comparative analysis. Orthop J Sports Med 7(3):2325967119831758. https://doi.org/10.1177/ 2325967119831758

70. Frank RM, Lee S, Bush-Joseph CA, Salata MJ, Mather RC 3rd, Nho SJ (2016) Outcomes for hip arthroscopy according to sex and age: a comparative matched-group analysis. J Bone Joint Surg Am 98(10):797-804. https://doi.org/10.2106/jbjs.15.00445

71. Frank RM, Ukwuani G, Clapp I, Chahla J, Nho SJ (2018) High rate of return to cycling after hip arthroscopy for femoroacetabular impingement syndrome. Sports Health 10(3):259-265. https://doi.org/10.1177/ 1941738117747851

72. Fukui K, Briggs KK, Trindade CA, Philippon MJ (2015) Outcomes after labral repair in patients with femoroacetabular impingement and borderline dysplasia. Arthroscopy 31(12):2371-2379. https://doi.org/10. 1016/j.arthro.2015.06.028

73. Fukui K, Trindade CA, Briggs KK, Philippon MJ (2015) Arthroscopy of the hip for patients with mild to moderate developmental dysplasia of the hip and femoroacetabular impingement: outcomes following hip arthroscopy for treatment of chondrolabral damage. Bone Joint J 97-b(10):1316-1321. https://doi.org/10.1302/0301-620x.97b10.35303

74. Ganz R, Parvizi J, Beck M, Leunig M, Notzli H, Siebenrock KA (2003) Femoroacetabular impingement: a cause for osteoarthritis of the hip. Clin Orthop Relat Res 417:112-120. https://doi.org/10.1097/01.blo. 0000096804.78689.c2

75. Gao F, Zhang B, Hu B, Lu M, An M, Fang Y, Zhao G, Shi C, Zhou J, Liu Y, Li C (2020) Outcomes of hip arthroscopy for femoroacetabular impingement in Chinese patients aged 50 years or older. Orthop Surg 12(3):843-851. https://doi.org/10.1111/os.12688

76. Gicquel T, Gédouin JE, Krantz N, May O, Gicquel P, Bonin N (2014) Function and osteoarthritis progression after arthroscopic treatment of femoro-acetabular impingement: a prospective study after a mean follow-up of 4.6 (4.2-5.5) years. Orthop Traumatol Surg Res 100(6):651656. https://doi.org/10.1016/j.otsr.2014.07.008

77. Gigi R, Rath E, Sharfman ZT, Shimonovich S, Ronen I, Amar E (2016) Hip arthroscopy for femoral-acetabular impingement: do active claims affect outcomes? Arthroscopy 32(4):595-600. https://doi.org/10.1016/j. arthro.2015.10.005

78. Grace T, Neumann J, Samaan MA, Souza RB, Majumdar S, LinkTM, Zhang AL (2018) Using the scoring hip osteoarthritis with magnetic resonance imaging (SHOMRI) system to assess intra-articular pathology in femoroacetabular impingement. J Orthop Res 36(11):3064-3070. https://doi.org/10.1002/jor.24102

79. Grace T, Samaan MA, Souza RB, Link TM, Majumdar S, Zhang AL (2018) Correlation of patient symptoms with labral and articular cartilage damage in femoroacetabular impingement. Orthop J Sports Med 6(6):2325967118778785. https://doi.org/10.1177/2325967118778785

80. Grant LF, Cooper DJ, Conroy JL (2017) The HAPI'Hip Arthroscopy prehabilitation intervention'study: does pre-habilitation affect outcomes in patients undergoing hip arthroscopy for femoro-acetabular impingement? J Hip Preserv Surg 4(1):85-92. https://doi.org/10.1093/jhps/ hnw046

81. Graves ML, Mast JW (2009) Femoroacetabular impingement: do outcomes reliably improve with surgical dislocations? Clin Orthop Relat Res 467(3):717-723. https://doi.org/10.1007/s11999-008-0648-y

82. Griffin DR, Dickenson EJ, O'Donnell J, Agricola R, Awan T, Beck M, Clohisy JC, Dijkstra HP, Falvey E, Gimpel M, Hinman RS, Holmich P, Kassarjian A, Martin HD, Martin R, Mather RC, Philippon MJ, Reiman MP, Takla A, Thorborg K, Walker S, Weir A, Bennell KL (2016) The Warwick Agreement 
on femoroacetabular impingement syndrome (FAl syndrome): an international consensus statement. Br J Sports Med 50(19):1169-1176. https://doi.org/10.1136/bjsports-2016-096743

83. Griffin DR, Dickenson EJ, Wall PDH, Achana F, Donovan JL, Griffin J, Hobson R, Hutchinson CE, Jepson M, Parsons NR, Petrou S, Realpe A, Smith J, Foster NE (2018) Hip arthroscopy versus best conservative care for the treatment of femoroacetabular impingement syndrome (UK FASHIoN): a multicentre randomised controlled trial. Lancet 391(10136):22252235. https://doi.org/10.1016/s0140-6736(18)31202-9

84. Griffin DR, Parsons N, Mohtadi NG, Safran MR, Multicenter Arthroscopy of the Hip Outcomes Research N (2012) A short version of the International Hip Outcome Tool (iHOT-12) for use in routine clinical practice. Arthroscopy 28(5):611-616. https://doi.org/10.1016/j.arthro.2012.02.027 (quiz 616-618)

85. Gupta A, Redmond JM, Hammarstedt JE, Lindner D, Stake CE, Domb BG (2015) Does obesity affect outcomes after hip arthroscopy? A cohort analysis. J Bone Joint Surg Am 97(1):16-23. https://doi.org/10.2106/ jbjs.n.00625

86. Gupta A, Redmond JM, Stake CE, Finch NA, Dunne KF, Domb BG (2014) Does the femoral cam lesion regrow after osteoplasty for femoroacetabular impingement? Two-year follow-up. Am J Sports Med 42(9):2149-2155. https://doi.org/10.1177/0363546514541782

87. Ha YC, Lim JY, Won YS, Lee YK, Koo KH, Kim JW (2020) Outcomes of arthroscopic femoroplasty in patients with cam lesions: minimum 2-year follow-up. J Orthop Surg 28(2):2309499020942049. https://doi. org/10.1177/2309499020942049

88. Hamula MJ, Ryan MK, Baron SL, Bloom DA, Youm T (2020) Atypical hip pain in femoroacetabular impingement: a comparison of outcomes based on primary hip pain location. Am J Sports Med 48(1):167-172. https://doi.org/10.1177/0363546519887733

89. Haskel JD, Baron SL, Zusmanovich M, Youm T (2020) Does concomitant lumbar spine disease adversely affect the outcomes of patients undergoing hip arthroscopy? Am J Sports Med 48(9):2178-2184. https://doi. org/10.1177/0363546520929344

90. Hassebrock JD, Krych AJ, Domb BG, Levy BA, Neville MR, Hartigan DE (2019) Bilateral hip arthroscopy: can results from initial arthroscopy for femoroacetabular impingement predict future contralateral results? Arthroscopy 35(6):1837-1844. https://doi.org/10.1016/j.arthro.2018.12. 033

91. Herrmann SJ, Bernauer M, Erdle B, Südkamp NP, Helwig P, Hauschild O (2016) Osteoarthritic changes rather than age predict outcome following arthroscopic treatment of femoroacetabular impingement in middle-aged patients. BMC Musculoskelet Disord 17:253. https://doi. org/10.1186/s12891-016-1108-6

92. Horisberger M, Brunner A, Herzog RF (2010) Arthroscopic treatment of femoral acetabular impingement in patients with preoperative generalized degenerative changes. Arthroscopy 26(5):623-629. https://doi.org/ 10.1016/j.arthro.2009.09.003

93. Humphrey JA, George MD, Bankes MJK (2018) Experience and outcome data of the British non-arthroplasty hip registry. Hip Int 28(4):429-433. https://doi.org/10.5301/hipint.5000594

94. Hwang JM, Hwang DS, Kang C, Lee WY, Lee GS, Lee JK, Kim YK (2019) Arthroscopic treatment for femoroacetabular impingement with extraspinal diffuse idiopathic skeletal hyperostosis. Clin Orthop Surg 11(3):275-281. https://doi.org/10.4055/cios.2019.11.3.275

95. Ilizaliturri VM Jr, Orozco-Rodriguez L, Acosta-Rodríguez E, CamachoGalindo J (2008) Arthroscopic treatment of cam-type femoroacetabular impingement: preliminary report at 2 years minimum follow-up. J Arthroplasty 23(2):226-234. https://doi.org/10.1016/j.arth.2007.03.016

96. Inan U, Harmanşa S, Ömeroğlu H (2016) Treatment of mixed type femoroacetabular impingement using safe surgical hip dislocation in adults. Eklem Hastalik Cerrahisi 27(3):160-166. https://doi.org/10.5606/ ehc.2016.32

97. Ishøi L, Thorborg K, Kraemer O, Hölmich P (2018) Return to sport and performance after hip arthroscopy for femoroacetabular impingement in 18- to 30-year-old athletes: a cross-sectional cohort study of 189 athletes. Am J Sports Med 46(11):2578-2587. https://doi.org/10.1177/ 0363546518789070

98. Ishøi L, Thorborg K, Kraemer O, Hölmich P (2019) The association between specific sports activities and sport performance following hip arthroscopy for femoroacetabular impingement syndrome: a secondary analysis of a cross-sectional cohort study including 184 athletes. J Hip Preserv Surg 6(2):124-133. https://doi.org/10.1093/jhps/ hnz017

99. Javed A, O'Donnell JM (2011) Arthroscopic femoral osteochondroplasty for cam femoroacetabular impingement in patients over 60 years of age. J Bone Joint Surg Br 93(3):326-331. https://doi.org/10.1302/0301620x.93b3.25262

100. Jochimsen KN, Magnuson JA, Kocan KR, Mattacola CG, Noehren B, Duncan ST, Jacobs CA (2019) Anxiety and depression are associated with lower preoperative quality of life and function but not duration of symptoms in patients with femoroacetabular impingement syndrome. J Hip Preserv Surg 6(3):207-213. https://doi.org/10.1093/jhps/hnz027

101. Jäger M, Bittersohl B, Zilkens C, Hosalkar HS, Stefanovska K, Kurth S, Krauspe R (2011) Surgical hip dislocation in symptomatic cam femoroacetabular impingement: what matters in early good results? Eur J Med Res 16(5):217-222. https://doi.org/10.1186/2047-783x-16-5-217

102. Kaldau NC, Brorson S, Hölmich P, Lund B (2018) Good midterm results of hip arthroscopy for femoroacetabular impingement. Dan Med J 65(6):A5483

103. Kaplan DJ, Samim M, Burke CJ, Baron SL, Meislin RJ, Youm T (2020) Decreased hip labral width measured via preoperative MRI is associated with inferior outcomes for arthroscopic labral repair for femoroacetabular impingement. Arthroscopy. https://doi.org/10.1016/j.arthro.2020.08. 006

104. Keating TC, Chahla J, Beck EC, Riff AJ, Clapp IM, Jan K, Nho SJ (2020) Return to pilates following hip arthroscopy for treatment of femoroacetabular impingement syndrome. J Hip Preserv Surg 6(4):339-345. https://doi.org/10.1093/jhps/hnz053

105. Kekatpure AL, Ahn T, Kim CH, Lee SJ, Yoon KS, Yoon PW (2017) Clinical outcomes of an initial 3-month trial of conservative treatment for femoroacetabular impingement. Indian J Orthop 51(6):681-686. https://doi. org/10.4103/ortho.IJOrtho_212_16

106. Khan M, Oduwole KO, Razdan P, Phillips M, Ekhtiari S, Horner NS, Samuelsson K, Ayeni OR (2016) Sources and quality of literature addressing femoroacetabular impingement: a scoping review 2011-2015. Curr Rev Musculoskelet Med 9(4):396-401. https://doi.org/10.1007/ s12178-016-9364-5

107. Kierkegaard S, Dalgas U, Lund B, Lipperts M, Søballe K, Mechlenburg I (2020) Despite patient-reported outcomes improve, patients with femoroacetabular impingement syndrome do not increase their objectively measured sport and physical activity level 1 year after hip arthroscopic surgery. Results from the HAFAI cohort. Knee Surg Sports Traumatol Arthrosc 28(5):1639-1647. https://doi.org/10.1007/ s00167-019-05503-5

108. Kierkegaard S, Mechlenburg I, Lund B, Rømer L, Søballe K, Dalgas U (2019) Is hip muscle strength normalised in patients with femoroacetabular impingement syndrome one year after surgery?: results from the HAFAl cohort. J Sci Med Sport 22(4):413-419. https://doi.org/10. 1016/j.jsams.2018.10.004

109. Kockara N, Sofu H, Issin A, Çamurcu Y, Bursali A (2018) Predictors of the clinical outcome and survival without degenerative arthritis after surgical treatment of femoroacetabular impingement. J Orthop Sci 23(1):117-121. https://doi.org/10.1016/j.jos.2017.09.002

110. Kouk S, Baron SL, Pham H, Campbell A, Begly J, Youm T (2020) Clinical outcomes of hip arthroscopy in patients with systemic inflammatory diseases compared with matched controls at a minimum of 2-year follow-up. Arthroscopy 36(5):1345-1352. https://doi.org/10.1016/j. arthro.2020.01.017

111. Krishnamoorthy VP, Beck EC, Kunze KN, Cancienne JM, Krivicich LM, Suppauksorn S, Ayeni OR, Nho SJ (2019) Radiographic prevalence of sacroiliac joint abnormalities and clinical outcomes in patients with femoroacetabular impingement syndrome. Arthroscopy 35(9):25982605.e2591. https://doi.org/10.1016/j.arthro.2019.03.030

112. Krishnamoorthy VP, Kunze KN, Beck EC, Cancienne JM, O'Keefe LS Ayeni OR, Nho SJ (2019) Radiographic prevalence of symphysis pubis abnormalities and clinical outcomes in patients with femoroacetabular impingement syndrome. Am J Sports Med 47(6):1467-1472. https:// doi.org/10.1177/0363546519837203

113. Krych AJ, King AH, Berardelli RL, Sousa PL, Levy BA (2016) Is subchondral acetabular edema or cystic change on MRI a contraindication for 
hip arthroscopy in patients with femoroacetabular impingement? Am J Sports Med 44(2):454-459. https://doi.org/10.1177/0363546515612448

114. Krych AJ, Thompson M, Knutson Z, Scoon J, Coleman SH (2013) Arthroscopic labral repair versus selective labral debridement in female patients with femoroacetabular impingement: a prospective randomized study. Arthroscopy 29(1):46-53. https://doi.org/10.1016/j.arthro. 2012.07.011

115. Kunze KN, Beck EC, Nwachukwu BU, Ahn J, Nho SJ (2019) Early hip arthroscopy for femoroacetabular impingement syndrome provides superior outcomes when compared with delaying surgical treatment beyond 6 months. Am J Sports Med 47(9):2038-2044. https://doi.org/ $10.1177 / 0363546519837192$

116. Kunze KN, Beck EC, Okoroha KR, Chahla J, Suppauksorn S, Bush-Joseph CA, Katakam A, Nho SJ (2019) Effect of prior ipsilateral lower extremity surgery on 2-year outcomes following hip arthroscopy for femoroacetabular impingement syndrome. J Hip Preserv Surg 6(3):241-248. https://doi.org/10.1093/jhps/hnz031

117. Kunze KN, Leong NL, Beck EC, Bush-Joseph CA, Nho SJ (2019) Hip arthroscopy for femoroacetabular impingement improves sleep quality postoperatively. Arthroscopy 35(2):461-469. https://doi.org/10.1016/j. arthro.2018.09.021

118. Lall AC, Secretov E, Battaglia MR, Maldonado DR, Perets I, Domb BG (2020) Effect of alcohol consumption on patient-reported outcomes in hip arthroscopy: a matched controlled study with minimum 2-year follow-up. Hip Int 30(4):457-468. https://doi.org/10.1177/1120700019 853554

119. Landis JR, Koch GG (1977) An application of hierarchical kappa-type statistics in the assessment of majority agreement among multiple observers. Biometrics 33(2):363-374

120. Lansdown DA, Kunze K, Ukwuani G, Waterman BR, Nho SJ (2018) The importance of comprehensive cam correction: radiographic parameters are predictive of patient-reported outcome measures at 2 years after hip arthroscopy. Am J Sports Med 46(9):2072-2078. https://doi. org/10.1177/0363546518780311

121. Lansdown DA, Ukwuani G, Kuhns B, Harris JD, Nho SJ (2018) Selfreported mental disorders negatively influence surgical outcomes after arthroscopic treatment of femoroacetabular impingement. Orthop J Sports Med 6(5):2325967118773312. https://doi.org/10.1177/23259 67118773312

122. Lee S, Frank RM, Harris J, Song SH, Bush-Joseph CA, Salata MJ, Nho SJ (2015) Evaluation of sexual function before and after hip arthroscopic surgery for symptomatic femoroacetabular impingement. Am J Sports Med 43(8):1850-1856. https://doi.org/10.1177/0363546515584042

123. Lerch S, Kasperczyk A, Berndt T, Rühmann O (2015) Ultrasonography can quantify the extent of osteochondroplasty after treatment of Cam-type femoroacetabular impingement. Int Orthop 39(5):853-858. https://doi.org/10.1007/s00264-014-2588-3

124. Levy DM, Cvetanovich GL, Kuhns BD, Greenberg MJ, Alter JM, Nho SJ (2017) Hip Arthroscopy for atypical posterior hip pain: a comparative matched-pair analysis. Am J Sports Med 45(7):1627-1632. https://doi. org/10.1177/0363546517692983

125. Lindman I, Öhlin A, Desai N, Samuelsson K, Ayeni OR, Hamrin Senorski E, Sansone M (2020) Five-year outcomes after arthroscopic surgery for femoroacetabular impingement syndrome in elite athletes. Am J Sports Med 48(6):1416-1422. https://doi.org/10.1177/0363546520908840

126. Lund B, Mygind-Klavsen B, Gronbech Nielsen T, Maagaard N, Kraemer O, Holmich P, Winge S, Lind M (2017) Danish Hip Arthroscopy Registry (DHAR): the outcome of patients with femoroacetabular impingement (FAI). J Hip Preserv Surg 4(2):170-177. https://doi.org/10.1093/jhps/ hnx009

127. Malagelada F, Del Carmen VA, Barke SJ, Guirao Cano L, Pleguezuelos Cobo E (2015) The anterior mini-open approach for femeroacetabular impingement: gait and functional assessment at one year post-surgery. Ann Phys Rehabil Med 58(2):60-65. https://doi.org/10.1016/j.rehab. 2014.09.013

128. Maldonado DR, Chen JW, Yelton MJ, Rosinsky PJ, Shapira J, Brayboy C, Lall AC, Domb BG (2020) Achieving successful outcomes of hip arthroscopy in the setting of generalized ligamentous laxity with labral preservation and appropriate capsular management: a propensity matched controlled study. Am J Sports Med 48(7):1625-1635. https:// doi.org/10.1177/0363546520914604
129. Malloy P, Stone AV, Kunze KN, Neal WH, Beck EC, Nho SJ (2019) Patients with unilateral femoroacetabular impingement syndrome have asymmetrical hip muscle cross-sectional area and compensatory muscle changes associated with preoperative pain level. Arthroscopy 35(5):1445-1453. https://doi.org/10.1016/j.arthro.2018.11.053

130. Mannion AF, Impellizzeri FM, Naal FD, Leunig M (2013) Fulfilment of patient-rated expectations predicts the outcome of surgery for femoroacetabular impingement. Osteoarthritis Cartilage 21(1):44-50. https://doi.org/10.1016/j.joca.2012.09.013

131. Mansell NS, Rhon DI, Meyer J, Slevin JM, Marchant BG (2018) Arthroscopic surgery or physical therapy for patients with femoroacetabular impingement syndrome: a randomized controlled trial with 2-year follow-up. Am J Sports Med 46(6):1306-1314. https://doi.org/10.1177/ 0363546517751912

132. Mardones R, Via AG, Rivera A, Tomic A, Somarriva M, Wainer M, Camacho D (2016) Arthroscopic treatment of femoroacetabular impingement in patients older than 60 years. Muscles Ligaments Tendons J 6(3):397-401. https://doi.org/10.11138/mltj/2016.6.3.397

133. Mardones R, Via AG, Tomic A, Rodriguez C, Salineros M, Somarriva M (2016) Arthroscopic release of iliopsoas tendon in patients with femoro-acetabular impingement: clinical results at mid-term follow-up. Muscles Ligaments Tendons J 6(3):378-383. https://doi.org/10.11138/ $\mathrm{mltj} / 2016.6 .3 .378$

134. Martin RL, Philippon MJ (2007) Evidence of validity for the hip outcome score in hip arthroscopy. Arthroscopy 23(8):822-826. https://doi.org/10. 1016/j.arthro.2007.02.004

135. Martínez D, Gómez-Hoyos J, Márquez W, Gallo J (2015) Factors associated with the failure of arthroscopic surgery treatment in patients with femoroacetabular impingement: a cohort study. Rev Esp Cir Ortop Traumatol 59(2):112-121. https://doi.org/10.1016/j.recot.2014.09.002

136. Mas Martinez J, Sanz-Reig J, Verdu Roman C, Suarez B, de Puga D, Martinez Gimenez E, Morales Santias M (2020) Recreational sports and intra-articular hip injuries in patients undergoing hip arthroscopy for femoroacetabular impingement. Arthrosc Sports Med Rehabil 2(4):e321-e328. https://doi.org/10.1016/j.asmr.2020.04.005

137. Matsuda DK, Burchette RJ (2013) Arthroscopic hip labral reconstruction with a gracilis autograft versus labral refixation: 2-year minimum outcomes. Am J Sports Med 41(5):980-987. https://doi.org/10.1177/ 0363546513482884

138. Matsuda DK, Gupta N, Khatod M, Matsuda NA, Anthony F, Sampson J, Burchette R (2017) Poorer arthroscopic outcomes of mild dysplasia with cam femoroacetabular impingement versus mixed femoroacetabular impingement in absence of capsular repair. Am J Orthop 46(1):E47-e53

139. Matsuda DK, Kivlan BR, Nho SJ, Wolff AB, Salvo JP Jr, Christoforetti JJ, Ellis TJ, Carreira DS (2019) Arthroscopic outcomes as a function of acetabular coverage from a large hip arthroscopy study group. Arthroscopy 35(8):2338-2345. https://doi.org/10.1016/j.arthro.2019.01.055

140. Menge TJ, Briggs KK, Dornan GJ, McNamara SC, Philippon MJ (2017) Survivorship and outcomes 10 years following hip arthroscopy for femoroacetabular impingement: labral debridement compared with labral repair. J Bone Joint Surg Am 99(12):997-1004. https://doi.org/10. 2106/jbjs. 16.01060

141. Mladenović D, Andjelković Z, Vukasinović Z, Mitković M, Milenković S, Micić I, Mladenović M (2014) Early clinical results of surgical treatment of patients with femoroacetabular impingement. Srp Arh Celok Lek 142(5-6):325-329. https://doi.org/10.2298/sarh1406325m

142. Moher D, Shamseer L, Clarke M, Ghersi D, Liberati A, Petticrew M, Shekelle P, Stewart LA, Group P-P (2015) Preferred reporting items for systematic review and meta-analysis protocols (PRISMA-P) 2015 statement. Syst Rev 4:1. https://doi.org/10.1186/2046-4053-4-1

143. Mohtadi NG, Griffin DR, Pedersen ME, Chan D, Safran MR, Parsons N, Sekiya JK, Kelly BT, Werle JR, Leunig M, McCarthy JC, Martin HD, Byrd JW, Philippon MJ, Martin RL, Guanche CA, Clohisy JC, Sampson TG, Kocher MS, Larson CM, Multicenter Arthroscopy of the Hip Outcomes Research N (2012) The development and validation of a self-administered quality-of-life outcome measure for young, active patients with symptomatic hip disease: the International Hip Outcome Tool (iHOT-33). Arthroscopy 28(5):595-605. https://doi.org/10.1016/j.arthro.2012.03.013 (quiz 606-510 e591)

144. Naal FD, Müller A, Varghese VD, Wellauer V, Impellizzeri FM, Leunig M (2017) Outcome of hip impingement surgery: does generalized joint 
hypermobility matter? Am J Sports Med 45(6):1309-1314. https://doi. org/10.1177/0363546516688636

145. Nabavi A, Olwill CM, Harris IA (2015) Preoperative predictors of outcome in the arthroscopic treatment of femoroacetabular impingement. Hip Int 25(5):402-405. https://doi.org/10.5301/hipint.5000261

146. Nakashima H, Utsunomiya H, Kanezaki S, Suzuki H, Nakamura E, Larson CM, Sakai A, Uchida S (2019) Is arthroscopic hip labral repair/reconstruction surgery effective for treating femoroacetabular impingement in the presence of osteoarthritis? Clin J Sport Med. https://doi.org/10. 1097/jsm.0000000000000768

147. Nawabi DH, Degen RM, Fields KG, McLawhorn A, Ranawat AS, Sink EL, Kelly BT (2016) Outcomes after arthroscopic treatment of femoroacetabular impingement for patients with borderline hip dysplasia. Am J Sports Med 44(4):1017-1023. https://doi.org/10.1177/0363546515 624682

148. Nepple JJ, Goljan P, Briggs KK, Garvey SE, Ryan M, Philippon MJ (2015) Hip strength deficits in patients with symptomatic femoroacetabular impingement and labral tears. Arthroscopy 31(11):2106-2111. https:// doi.org/10.1016/j.arthro.2015.04.095

149. Nepple JJ, Zebala LP, Clohisy JC (2009) Labral disease associated with femoroacetabular impingement: do we need to correct the structural deformity? J Arthroplasty 24(6 Suppl):1 14-119. https://doi.org/10. 1016/j.arth.2009.06.003

150. Nho SJ, Beck EC, Nwachukwu BU, Cvetanovich GL, Neal WH, Harris JD, Weber AE, Mather RC (2019) Survivorship and outcome of hip arthroscopy for femoroacetabular impingement syndrome performed with modern surgical techniques. Am J Sports Med 47(7):1662-1669. https:// doi.org/10.1177/0363546519843936

151. Nwachukwu BU, Beck EC, Lee EK, Cancienne JM, Waterman BR, Paul K Nho SJ (2020) Application of machine learning for predicting clinically meaningful outcome after arthroscopic femoroacetabular impingement surgery. Am J Sports Med 48(2):415-423. https://doi.org/10.1177/ 0363546519892905

152. Nwachukwu BU, Chang B, Adjei J, Schairer WW, Ranawat AS, Kelly BT, Nawabi DH (2018) Time required to achieve minimal clinically important difference and substantial clinical benefit after arthroscopic treatment of femoroacetabular impingement. Am J Sports Med 46(11):2601-2606. https://doi.org/10.1177/0363546518786480

153. Nwachukwu BU, Fields K, Chang B, Nawabi DH, Kelly BT, Ranawat AS (2017) Preoperative outcome scores are predictive of achieving the minimal clinically important difference after arthroscopic treatment of femoroacetabular impingement. Am J Sports Med 45(3):612-619. https://doi.org/10.1177/0363546516669325

154. Nwachukwu BU, Rebolledo BJ, McCormick F, Rosas S, Harris JD, Kelly BT (2016) Arthroscopic versus open treatment of femoroacetabular impingement: a systematic review of medium- to long-term outcomes. Am J Sports Med 44(4):1062-1068. https://doi.org/10.1177/0363546515 587719

155. Ohlin A, Karlsson L, Senorski EH, Jonasson P, Ahlden M, Baranto A, Ayeni OR, Sansone M (2019) Quality assessment of prospective cohort studies evaluating arthroscopic treatment for femoroacetabular impingement syndrome: a systematic review. Orthop J Sports Med 7(5):2325967119838533. https://doi.org/10.1177/2325967119838533

156. Palmer AJR, Ayyar Gupta V, Fernquest S, Rombach I, Dutton SJ, Mansour R, Wood S, Khanduja V, Pollard TCB, McCaskie AW, Barker KL, Andrade T, Carr AJ, Beard DJ, Glyn-Jones S (2019) Arthroscopic hip surgery compared with physiotherapy and activity modification for the treatment of symptomatic femoroacetabular impingement: multicentre randomised controlled trial. BMJ 364:1185. https://doi.org/10.1136/bmj.l185

157. Park MS, Yoon SJ, Kim YJ, Chung WC (2014) Hip arthroscopy for femoroacetabular impingement: the changing nature and severity of associated complications over time. Arthroscopy 30(8):957-963. https://doi. org/10.1016/j.arthro.2014.03.017

158. Patrick DL, Burke LB, Powers JH, Scott JA, Rock EP, Dawisha S, O'Neill R, Kennedy DL (2007) Patient-reported outcomes to support medical product labeling claims: FDA perspective. Value Health 10(Suppl 2):S125-137. https://doi.org/10.1111/j.1524-4733.2007.00275.x

159. Perets I, Chaharbakhshi EO, Mu B, Ashberg L, Battaglia MR, Yuen LC, Domb BG (2018) Hip arthroscopy in patients ages 50 years or older: minimum 5-year outcomes, survivorship, and risk factors for conversion to total hip replacement. Arthroscopy 34(11):3001-3009. https://doi. org/10.1016/j.arthro.2018.05.034

160. Perets I, Prat D, Close MR, Chaharbakhshi EO, Rabe SM, Battaglia MR, Domb BG (2019) Patients undergoing hip arthroscopy with active workers' compensation claims do not demonstrate inferior outcomes at mid-term. Hip Int 29(5):543-549. https://doi.org/10.1177/1120700018 810537

161. Perets I, Rybalko D, Chaharbakhshi EO, Mu BH, Chen AW, Domb BG (2018) Minimum five-year outcomes of hip arthroscopy for the treatment of femoroacetabular impingement and labral tears in patients with obesity: a match-controlled study. J Bone Joint Surg Am 100(11):965-973. https://doi.org/10.2106/jbjs.17.00892

162. Philippon MJ, Briggs KK, Yen YM, Kuppersmith DA (2009) Outcomes following hip arthroscopy for femoroacetabular impingement with associated chondrolabral dysfunction: minimum two-year follow-up. J Bone Joint Surg Br 91(1):16-23. https://doi.org/10.1302/0301-620x. 91b1.21329

163. Philippon MJ, Schroder ESBG, Briggs KK (2012) Hip arthroscopy for femoroacetabular impingement in patients aged 50 years or older. Arthroscopy 28(1):59-65. https://doi.org/10.1016/j.arthro.2011.07.004

164. Philippon MJ, Weiss DR, Kuppersmith DA, Briggs KK, Hay CJ (2010) Arthroscopic labral repair and treatment of femoroacetabular impingement in professional hockey players. Am J Sports Med 38(1):99-104. https://doi.org/10.1177/0363546509346393

165. Polesello GC, Cinagawa EH, Cruz PD, de Queiroz MC, Borges CJ, Junior WR, Daniachi D, Guimarães RP, Honda EK, Ono NK (2012) Surgical treatment for femoroacetabular impingement in a group that performs squats. Rev Bras Ortop 47(4):488-492. https://doi.org/10.1016/s22554971(15)30134-8

166. Polesello GC, Queiroz MC, Ono NK, Honda EK, Guimarāes RP, Junior WR (2009) Arthroscopic treatment of femoroacetabular impingement. Rev Bras Ortop 44(3):230-238. https://doi.org/10.1016/s2255-4971(15) 30073-2

167. Potter MQ, Wylie JD, Sun GS, Beckmann JT, Aoki SK (2014) Psychologic distress reduces preoperative self-assessment scores in femoroacetabular impingement patients. Clin Orthop Relat Res 472(6):1886-1892. https://doi.org/10.1007/s11999-014-3531-z

168. Przybyl M, Walenczak K, Domzalski ME (2018) Athletes do better after FAl arthroscopic treatment in male population. J Orthop Surg 26(1):2309499018760111. https://doi.org/10.1177/2309499018760111

169. Ragab R, Elkhadrawe T, Housden P, Abotaleb A (2018) Results of arthroscopic treatment of femroacetabular impingement (FAl). Alex J Med 54(4):361-363. https://doi.org/10.1016/j.ajme.2018.04.002

170. Ramisetty N, Kwon Y, Mohtadi N (2015) Patient-reported outcome measures for hip preservation surgery-a systematic review of the literature. J Hip Preserv Surg 2(1):15-27. https://doi.org/10.1093/jhps/hnv002

171. Ramos N, Gerhardt M, Banffy M (2020) Hip arthroscopy for femoroacetabular impingement (FAl) patients with self-reported allergies: do multiple allergies have an effect on outcome? J Hip Preserv Surg 6(4):346-352. https://doi.org/10.1093/jhps/hnz045

172. Ramos N, Youssefzadeh K, Gerhardt M, Banffy M (2020) Results of hip arthroscopy in elite level water polo players with femoroacetabular impingement: return to play and patient satisfaction. J Hip Preserv Surg 7(1):116-121. https://doi.org/10.1093/jhps/hnz069

173. Redmond JM, El Bitar YF, Gupta A, Stake CE, Vemula SP, Domb BG (2015) Arthroscopic acetabuloplasty and labral refixation without labral detachment. Am J Sports Med 43(1):105-112. https://doi.org/10.1177/ 0363546514555330

174. Rego PA, Mascarenhas V, Oliveira FS, Pinto PC, Sampaio E, Monteiro $J$ (2018) Arthroscopic versus open treatment of cam-type femoroacetabular impingement: retrospective cohort clinical study. Int Orthop 42(4):791-797. https://doi.org/10.1007/s00264-017-3735-4

175. Reiman MP, Peters S, Sylvain J, Hagymasi S, Ayeni OR (2018) Prevalence and consistency in surgical outcome reporting for femoroacetabular impingement syndrome: a scoping review. Arthroscopy 34(4):13191328.e1319. https://doi.org/10.1016/j.arthro.2017.11.037

176. Ribas M, Marín-Peña OR, Regenbrecht B, De La Torre B, Vilarrubias JM (2007) Hip osteoplasty by an anterior minimally invasive approach for active patients with femoroacetabular impingement. Hip Int 17(2):91-98. https://doi.org/10.5301/hip.2008.4268 
177. Riff AJ, Ukwuani G, Clapp I, Movassaghi K, Kelly DM, Nho SJ (2018) High rate of return to high-intensity interval training after arthroscopic management of femoroacetabular impingement syndrome. Am J Sports Med 46(11):2594-2600. https://doi.org/10.1177/0363546518776638

178. Rivera E, Seijas R, Rubio M, García-Balletbó M, Vilar JM, Boada PL, Cugat R (2020) Outcomes at 2-years follow-up after hip arthroscopy combining bone marrow concentrate. J Invest Surg 33(7):655-663. https://doi. org/10.1080/08941939.2018.1535010

179. Roos BD, Roos MV, Camisa Júnior A, Lima EMU, Betto MD (2017) Open versus arthroscopic approach in the treatment of femoroacetabular impingement: a case-control study with two-years follow up. Rev Bras Ortop 52(Suppl 1):21-28. https://doi.org/10.1016/j.rboe.2017.07.007

180. Roos BD, Roos MV, Júnior AC, Lima EM, Gyboski DP, Martins LS (2015) Extracapsular approach for arthroscopic treatment of femoroacetabular impingement: clinical and radiographic results and complications. Rev Bras Ortop 50(4):430-437. https://doi.org/10.1016/j.rboe.2015.06.011

181. Rylander JH, Shu B, Andriacchi TP, Safran MR (2011) Preoperative and postoperative sagittal plane hip kinematics in patients with femoroacetabular impingement during level walking. Am J Sports Med 39(Suppl):36s-42s. https://doi.org/10.1177/0363546511413993

182. Saltzman BM, Kuhns BD, Basques B, Leroux T, Alter J, Mather RC 3rd, Salata MJ, Nho SJ (2017) The influence of body mass index on outcomes after hip arthroscopic surgery with capsular plication for the treatment of femoroacetabular impingement. Am J Sports Med 45(10):2303-2311. https://doi.org/10.1177/0363546517705617

183. Samaan MA, Grace T, Zhang AL, Majumdar S, Souza RB (2020) Short term outcomes of hip arthroscopy on hip joint mechanics and cartilage health in patients with femoroacetabular impingement syndrome. Clin Biomech 71:214-220. https://doi.org/10.1016/j.clinbiomech.2019.11. 014

184. Sanders TL, Reardon P, Levy BA, Krych AJ (2017) Arthroscopic treatment of global pincer-type femoroacetabular impingement. Knee Surg Sports Traumatol Arthrosc 25(1):31-35. https://doi.org/10.1007/ s00167-016-4266-z

185. Sansone $M$, Ahlden $M$, Jonasson $P$, Thomee $C$, Sward L, Baranto A, Karlsson J, Thomee R (2014) A Swedish hip arthroscopy registry: demographics and development. Knee Surg Sports Traumatol Arthrosc 22(4):774-780. https://doi.org/10.1007/s00167-014-2840-9

186. Sansone M, Ahldén M, Jonasson P, Thomeé C, Swärd L, Baranto A, Karlsson J, Thomeé R (2015) Good results after hip arthroscopy for femoroacetabular impingement in top-level athletes. Orthop J Sports Med 3(2):2325967115569691. https://doi.org/10.1177/2325967115 569691

187. Sansone M, Ahldén M, Jonasson P, Thomeé C, Swärd L, Collin D, Baranto A, Karlsson J, Thomeé R (2016) Outcome of hip arthroscopy in patients with mild to moderate osteoarthritis-A prospective study. J Hip Preserv Surg 3(1):61-67. https://doi.org/10.1093/jhps/hnv079

188. Sansone M, Ahldén M, Jónasson P, Thomeé C, Swärd L, Öhlin A, Baranto A, Karlsson J, Thomeé R (2017) Outcome after hip arthroscopy for femoroacetabular impingement in 289 patients with minimum 2-year follow-up. Scand J Med Sci Sports 27(2):230-235. https://doi.org/10. 1111/sms.12641

189. Sariali E, Vandenbulcke F (2018) Clinical outcomes following arthroscopic treatment of femoro-acetabular impingement using a minimal traction approach and an initial capsulotomy. Minimum two year follow-up. Int Orthop 42(11):2549-2554. https://doi.org/10.1007/ s00264-018-3904-0

190. Scanaliato JP, Christensen DL, Salfiti C, Herzog MM, Wolff AB (2018) Primary circumferential acetabular labral reconstruction: achieving outcomes similar to primary labral repair despite more challenging patient characteristics. Am J Sports Med 46(9):2079-2088. https://doi.org/10. $1177 / 0363546518775425$

191. Shaw KA, Jacobs JM, Evanson JR, Pniewski J, Dickston ML, Mueller T, Bojescul JA (2017) Functional outcomes of hip arthroscopy in an active duty military population utilizing a criterion-based early weight bearing progression. Int J Sports Phys Ther 12(5):840-847

192. Shibata KR, Matsuda S, Safran MR (2017) Arthroscopic hip surgery in the elite athlete: comparison of female and male competitive athletes. Am J Sports Med 45(8):1730-1739. https://doi.org/10.1177/0363546517 697296
193. Sim Y, Horner NS, de Sa D, Simunovic N, Karlsson J, Ayeni OR (2015) Reporting of non-hip score outcomes following femoroacetabular impingement surgery: a systematic review. J Hip Preserv Surg 2(3):224-241. https://doi.org/10.1093/jhps/hnv048

194. Skendzel JG, Philippon MJ, Briggs KK, Goljan P (2014) The effect of joint space on midterm outcomes after arthroscopic hip surgery for femoroacetabular impingement. Am J Sports Med 42(5):1127-1133. https:// doi.org/10.1177/0363546514526357

195. Skowronek P, Synder M, Polguj M, Marczak D, Sibiński M (2017) Treatment of femoroacetabular impingement with a mini-open direct anterior approach. Indian J Orthop 51(6):677-680. https://doi.org/10. 4103/ortho.IJOrtho_248_16

196. Smith-Petersen MN (2009) The classic: treatment of malum coxae senilis, old slipped upper femoral epiphysis, intrapelvic protrusion of the acetabulum, and coxa plana by means of acetabuloplasty. 1936. Clin Orthop Relat Res 467(3):608-615. https://doi.org/10.1007/ s11999-008-0670-0

197. Sochacki KR, Brown L, Cenkus K, Di Stasi S, Harris JD, Ellis TJ (2018) Preoperative depression is negatively associated with function and predicts poorer outcomes after hip arthroscopy for femoroacetabular impingement. Arthroscopy 34(8):2368-2374. https://doi.org/10.1016/j. arthro.2018.03.020

198. Sochacki KR, Jack RA 2nd, Bekhradi A, Delgado D, McCulloch PC, Harris JD (2018) Are self-reported medication allergies associated with worse hip outcome scores prior to hip arthroscopy? Arthroscopy 34(6):18561861. https://doi.org/10.1016/j.arthro.2018.01.025

199. Spencer-Gardner L, Dissanayake R, Kalanie A, Singh P, O'Donnell J (2017) Hip arthroscopy results in improved patient reported outcomes compared to non-operative management of waitlisted patients. J Hip Preserv Surg 4(1):39-44. https://doi.org/10.1093/jhps/hnw051

200. Srinivasan SC, Hosny HA, Williams MR (2013) Combined hip arthroscopy and limited open osteochondroplasty for anterior femoroacetabular impingement: early patient reported outcomes. Hip Int 23(2):218-224. https://doi.org/10.5301/hip.2013.10728

201. Stone AV, Beck EC, Malloy P, Chahla J, Nwachukwu BU, Neal WH, Nho SJ (2019) Preoperative predictors of achieving clinically significant athletic functional status after hip arthroscopy for femoroacetabular impingement at minimum 2-year follow-up. Arthroscopy 35(11):3049-3056. e3041. https://doi.org/10.1016/j.arthro.2019.05.022

202. Stone AV, Malloy P, Beck EC, Neal WH, Waterman BR, Bush-Joseph CA, Nho SJ (2019) Predictors of persistent postoperative pain at minimum 2 years after arthroscopic treatment of femoroacetabular impingement. Am J Sports Med 47(3):552-559. https://doi.org/10.1177/0363546518 817538

203. Stähelin L, Stähelin T, Jolles BM, Herzog RF (2008) Arthroscopic offset restoration in femoroacetabular cam impingement: accuracy and early clinical outcome. Arthroscopy 24(1):51-57.e51. https://doi.org/10. 1016/j.arthro.2007.08.010

204. Thomas DD, Bernhardson AS, Bernstein E, Dewing CB (2017) Hip arthroscopy for femoroacetabular impingement in a military population. Am J Sports Med 45(14):3298-3304. https://doi.org/10.1177/03635 46517726984

205. Thorborg K, Holmich P, Christensen R, Petersen J, Roos EM (2011) The Copenhagen Hip and Groin Outcome Score (HAGOS): development and validation according to the COSMIN checklist. Br J Sports Med 45(6):478-491. https://doi.org/10.1136/bjsm.2010.080937

206. Thorborg K, Tijssen M, Habets B, Bartels EM, Roos EM, Kemp J, Crossley KM, Holmich P (2015) Patient-Reported Outcome (PRO) questionnaires for young to middle-aged adults with hip and groin disability: a systematic review of the clinimetric evidence. Br J Sports Med 49(12):812. https://doi.org/10.1136/bjsports-2014-094224

207. Tjong VK, Cogan CJ, Riederman BD, Terry MA (2016) A qualitative assessment of return to sport after hip arthroscopy for femoroacetabular impingement. Orthop J Sports Med 4(11):2325967116671940. https:// doi.org/10.1177/2325967116671940

208. Vahedi H, Aalirezaie A, Schlitt PK, Parvizi J (2019) Acetabular retroversion is a risk factor for less optimal outcome after femoroacetabular impingement surgery. J Arthroplasty 34(7):1342-1346. https://doi.org/ 10.1016/j.arth.2019.02.050 
209. Wadhwani J, Correa BP, Chicote HH (2018) Arthroscopic aproach of femoroacetabular impigement: early clinical outcomes. A multicentric study. J Orthop 15(3):754-756. https://doi.org/10.1016/j.jor.2018.05.044

210. Westermann RW, Hu J, Hagen MS, Willey M, Lynch TS, Rosneck J (2018) Epidemiology and detrimental impact of opioid use in patients undergoing arthroscopic treatment of femoroacetabular impingement syndrome. Arthroscopy 34(10):2832-2836. https://doi.org/10.1016/j. arthro.2018.06.038

211. Wu CT, Mahameed M, Lin PC, Lu YD, Kuo FC, Lee MS (2019) Treatment of cam-type femoroacetabular impingement using anterolateral mini-open and arthroscopic osteochondroplasty. J Orthop Surg Res 14(1):222. https://doi.org/10.1186/s13018-019-1257-z

212. Wörner T, Nilsson J, Thorborg K, Granlund V, Stålman A, Eek F (2019) Hip function 6 to 10 months after arthroscopic surgery: a cross-sectional comparison of subjective and objective hip function, including performance-based measures, in patients versus controls. Orthop J Sports Med 7(6):2325967119844821. https://doi.org/10.1177/23259 67119844821

213. Yeung M, Khan M, Schreiber VM, Adamich J, Letkemann S, Simunovic N, Bhandari M, MusahI V, Philippon MJ, Safran MR, Ayeni OR (2014) Global discrepancies in the diagnosis, surgical management, and investigation of femoroacetabular impingement. Arthroscopy 30(12):1625-1633. https://doi.org/10.1016/j.arthro.2014.06.008

214. Yoo Jl, Ha YC, Lee YK, Lee GY, Yoo MJ, Koo KH (2017) Morphologic changes and outcomes after arthroscopic acetabular labral repair evaluated using postoperative computed tomography arthrography. Arthroscopy 33(2):337-345. https://doi.org/10.1016/j.arthro.2016.08.022

215. Yun HH, Shon WY, Yun JY (2009) Treatment of femoroacetabular impingement with surgical dislocation. Clin Orthop Surg 1(3):146-154. https://doi.org/10.4055/cios.2009.1.3.146

216. Zhou J, Melugin HP, Hale RF, Leland DP, Bernard CD, Levy BA, Krych AJ (2020) The prevalence of radiographic findings of structural hip deformities for femoroacetabular impingement in patients with hip pain. Am J Sports Med 48:647-653. https://doi.org/10.1177/0363546519896355

217. Zhu X (2020) Efficacy of preemptive analgesia versus postoperative analgesia of celecoxib on postoperative pain, patients' global assessment and hip function recovery in femoroacetabular impingement patients underwent hip arthroscopy surgery. Inflammopharmacology 28(1):131-137. https://doi.org/10.1007/s10787-019-00648-8

218. Zimmerer A, Bock M, Hoffmann M, Miehlke W, Sobau C (2018) Return to work after arthroscopic surgery for femoroacetabular impingement in patients younger than 30 years (Return-to-Work" nach arthroskopischer FAl-Chirurgie bei Patienten unter 30 Jahren.). Sports Orthop Traumatol 34(1):31-37. https://doi.org/10.1016/j.orthtr.2017.10.006

219. Zusmanovich M, Thompson K, Campbell A, Youm T (2020) Outcomes of preoperative opioid usage in hip arthroscopy: a comparison to opioid naïve patients. Arthroscopy. https://doi.org/10.1016/j.arthro.2020.06 005

220. Öhlin A, Sansone M, Ayeni OR, Swärd L, Ahldén M, Baranto A, Karlsson $J$ (2017) Predictors of outcome at 2-year follow-up after arthroscopic treatment of femoro-acetabular impingement. J Hip Preserv Surg 4(3):224-230. https://doi.org/10.1093/jhps/hn×016

\section{Publisher's Note}

Springer Nature remains neutral with regard to jurisdictional claims in published maps and institutional affiliations.

\section{Submit your manuscript to a SpringerOpen ${ }^{\circ}$ journal and benefit from:}

- Convenient online submission

- Rigorous peer review

- Open access: articles freely available online

- High visibility within the field

- Retaining the copyright to your article

Submit your next manuscript at $\boldsymbol{\nabla}$ springeropen.com 